\title{
From Weak to Strong Zero-Knowledge and Applications ${ }^{\star}$
}

\author{
Kai-Min Chung ${ }^{1, \star \star}$, Edward Lui ${ }^{2}$, and Rafael Pass ${ }^{2, \star \star \star}$ \\ 1 Academia Sinica, Taiwan \\ kmchung@iis.sinica.edu.tw \\ 2 Cornell University, USA \\ \{luied,rafael\}@cs. cornell.edu
}

\begin{abstract}
The notion of zero-knowledge 20] is formalized by requiring that for every malicious efficient verifier $V^{*}$, there exists an efficient simulator $S$ that can reconstruct the view of $V^{*}$ in a true interaction with the prover, in a way that is indistinguishable to every polynomial-time distinguisher. Weak zero-knowledge weakens this notions by switching the order of the quantifiers and only requires that for every distinguisher $D$, there exists a (potentially different) simulator $S_{D}$.

In this paper we consider various notions of zero-knowledge, and investigate whether their weak variants are equivalent to their strong variants. Although we show (under complexity assumption) that for the standard notion of zero-knowledge, its weak and strong counterparts are not equivalent, for meaningful variants of the standard notion, the weak and strong counterparts are indeed equivalent. Towards showing these equivalences, we introduce new non-black-box simulation techniques permitting us, for instance, to demonstrate that the classical 2-round graph non-isomorphism protocol of Goldreich-Micali-Wigderson [18] satisfies a "distributional" variant of zero-knowledge.

Our equivalence theorem has other applications beyond the notion of zero-knowledge. For instance, it directly implies the dense model theorem of Reingold et al (STOC '08), and the leakage lemma of Gentry-Wichs (STOC '11), and provides a modular and arguably simpler proof of these results (while at the same time recasting these result in the language of zero-knowledge).
\end{abstract}

* A full version of this paper is available at https://eprint.iacr.org/2013/260

** Chung is supported in part by NSF Award CNS-1217821, NSF Award CCF1214844, Pass' Sloan Fellowship, and Ministry of Science and Technology MOST 103-2221-E-001-022-MY3; part of this work was done while being at Cornell University.

${ }^{\star \star \star}$ Pass is supported in part by an Alfred P. Sloan Fellowship, Microsoft New Faculty Fellowship, NSF CAREER Award CCF-0746990, NSF Award CCF-1214844, NSF Award CNS-1217821, AFOSR YIP Award FA9550-10-1-0093, and DARPA and AFRL under contract FA8750-11-2-0211. 


\section{Introduction}

The notion of zero-knowledge, and the simulation-paradigm used to define it, is of fundamental importance in modern cryptography - most definitions of protocol security rely on it. In a zero-knowledge protocol, a prover $P$ can convince a verifier $V$ of the validity of some mathematical statement $x \in L$, while revealing "zero (additional) knowledge" to $V$. This zero-knowledge property is formalized by requiring that for every potentially malicious efficient verifier $V^{*}$, there exists an efficient simulator $S$ that, without talking to $P$, is able to "indistinguishably reconstruct" the view of $V^{*}$ in a true interaction with $P$. The traditional way of defining what it means to "indistinguishably reconstruct" is to require that the output of $S$ cannot be distinguished (with more than negligible probability) from the true view of $V^{*}$ by any efficient distinguisher $D$; that is, we have a universal simulator that works for all distinguishers $D$.

A seemingly weaker way to define the zero-knowledge property is to require that for every distinguisher $D$, there exists a "distinguisher-dependent" simulator $S_{D}$ such that the output of $S_{D}$ cannot be distinguished from the true view of $V^{*}$ by the particular distinguisher $D$; following [12, we refer to this weaker notion of zero-knowledge as weak zero-knowledge.

The main question addressed in this paper is whether this switch in the order of the quantifiers yields an equivalent notion. More specifically, we consider various notions of zero-knowledge, and investigate whether their weak (distinguisherdependent simulator) variants are equivalent to their strong (universal simulator) variants. Towards addressing this question, we introduce new non-black-box simulation techniques permitting us, for instance, to demonstrate that the classical 2-round graph non-isomorphism protocol of Goldreich-Micali-Wigderson [18] satisfies a "distributional" variant of zero-knowledge. Our results also reveal deep connections between the notion of zero-knowledge and the dense model theorem of Reingold et al 28] (which in turn is related to questions such as the XOR Lemma 32 and Szemeredi's regularity lemma [15]; see [30] for more details).

\subsection{From Weak to Strong Zero-Knowledge}

Our first result shows that (under plausible complexity-theoretic assumptions) for the standard definition of zero-knowledge, weak zero-knowledge is a strictly weaker requirement than (strong) zero-knowledge.

Theorem 1 (Informally stated). Assume the existence of "timed commitments" and "timed one-way permutations". Then, there exists an interactive proof for a language $L \in \mathrm{NP}$ that is weak zero-knowledge but not (strong) zeroknowledge.

Motivated by this separation, we turn to consider relaxed notions of zeroknowledge. We first consider a concrete security variant of the notion of zeroknowledge. Roughly speaking, we call a protocol $(t, \epsilon)$-zero-knowledge if the zero-knowledge property holds with respect to all $t(n)$-time bounded distinguishers (as opposed to all polynomial-time distinguishers), and we require that the 
distinguishability gap is bounded by $\epsilon(n)$ (as opposed to being negligible), where $n$ is the length of the statement $x$ being proved. Weak $(t, \epsilon)$-zero-knowledge is defined analogously (by again switching the order of the quantifiers).

Note that if $(P, V)$ is $(t, \epsilon)$-zero-knowledge (resp. weak $(t, \epsilon)$-zero-knowledge) for some super-polynomial function $t$ and some negligible function $\epsilon$, then $(P, V)$ is zero-knowledge (resp. weak zero-knowledge) in the classic sense. We here consider a slightly relaxed notion where we only require $(P, V)$ to be $(t, \epsilon)$-zeroknowledge for all polynomials $t$ and all inverse polynomials $\epsilon$. (Note that this is weaker than the standard definition of zero-knowledge since now the runningtime of the simulator may depend on the bounds $t$ and $\epsilon$.) Perhaps surprisingly, we show that for this relaxed notion of zero-knowledge, the weak and strong versions lead to an equivalent definition.

Theorem 2 (Informally stated). If an interactive proof $(P, V)$ is weak $(t, \epsilon)$-zero knowledge for every polynomialt and every inverse polynomial $\epsilon$, then $(P, V)$ is also $\left(t^{\prime}, \epsilon^{\prime}\right)$-zero knowledge for every polynomial $t^{\prime}$ and every inverse polynomial $\epsilon^{\prime}$.

We highlight that the "universal" simulator $S$ constructed in the proof of Theorem 2 makes use of the malicious verifier $V^{*}$ in a non-black-box way. On a very high-level (and significantly oversimplifying), the idea behind Theorem 2 is to rely on Von Neumann's minimax theorem to obtain the universal simulator from the "distinguisher-dependent" simulators; the non-black-box nature of the universal simulator comes from the fact that defining the "utility function" we use with the minimax theorem requires knowing the auxiliary inputs received by $V^{*}$, and thus we make non-black-box use of $V^{*}$.

Implementing this approach becomes quite non-trivial since we require the existence of a uniform polynomial-time simulator for every uniform polynomialtime verifier - the minimax theorem only guarantees the existence of a distribution over polynomial-time machines that simulates the view of the verifier, but it is not clear if this distribution can be computed in uniform polynomial time. We overcome this issue by instead relying on a multiplicative weights algorithm to appropriately implement an approximate minimax strategy; see Section 1.4 for more details.

\subsection{From Super-Weak to Strong Distributional Zero-Knowledge}

Note that although in the definition of weak zero-knowledge the simulator may depend on the distinguisher, we still require that the probability that the distinguisher outputs 1 when given the output of the simulator is close to the probability that the distinguisher outputs 1 when given the true view of the malicious verifier $V^{*}$. An even weaker condition (considered in 21]) only requires that the simulator manages to make the distinguisher output 1 with at least as high probability (minus some "small" gap) as the probability that the distinguisher outputs 1 when given a true view of $V^{*}$. That is, we only consider "one-sided" indistinguishability. We refer to such a zero-knowledge property as super-weak zero-knowledge. 
It is not hard to see that super-weak $(t, \epsilon)$-zero-knowledge is not equivalent to weak $(t, \epsilon)$-zero-knowledge (see the full version of this paper for the proof). Thus, we here consider an alternative "distributional" notion of zero-knowledge (a la [17]) where indistinguishability of the simulation is only required for any distribution over statements (and auxiliary inputs), and the simulator as well as the distinguisher can depend on the distribution. Additionally, we here model both the distinguisher and the simulator as non-uniform polynomial-time algorithms (as opposed to uniform ones). (The combination of these variants was previously considered by 12, 1) We refer to such a notion of zero-knowledge as distributional zero-knowledge, and analogously define distributional $(t, \epsilon)$-zero-knowledge as well as weak (resp. super-weak) distributional $(t, \epsilon)$-zero-knowledge. Roughly speaking, distributional zero-knowledge captures the intuition that proofs of "random" statements do not provide the verifier with any new knowledge (beyond the statement proved). Perhaps surprisingly, we show that super-weak distributional $(t, \epsilon)$-zero-knowledge is equivalent to (strong) distributional $(t, \epsilon)$ zero-knowledge if we consider all polynomials $t$ and all inverse polynomials $\epsilon$.

Theorem 3 (Informally stated). If an interactive proof $(P, V)$ is super-weak distributional $(t, \epsilon)$-zero-knowledge for every polynomial $t$ and every inverse polynomial $\epsilon$, then $(P, V)$ is also distributional $\left(t^{\prime}, \epsilon^{\prime}\right)$-zero knowledge for every polynomial $t^{\prime}$ and every inverse polynomial $\epsilon^{\prime}$.

In contrast to Theorem 22 the proof of Theorem 3 follows from a rather direct use of the minimax theorem; see Section 1.4 for more details. We also show that any protocol where the prover is "laconic" [19] - that is, it sends only $O(\log n)$ bits in total, is super-weak (distributional) zero-knowledge; combining this result with Theorem 3 thus yields the following theorem.

Theorem 4 (Informally stated). Let $(P, V)$ be an interactive proof with a laconic prover for a language $L$. Then $(P, V)$ is distributional $(t, \epsilon)$-zero-knowledge for every polynomial $t$ and every inverse polynomial $\epsilon$.

Given Theorem 3, the proof of Theorem 4 is very straight-forward: to show that laconic proofs are super-weak zero-knowledge, have the simulator simply enumerate all possible prover messages and keep the one that the distinguisher "likes the most" (i.e., makes the distinguisher output 1 with as high probability as possible); note that we here rely crucially on the fact that we only need to achieve "one-sided" indistinguishability.

Theorem 4 may seem contradictory. An interactive proof with a laconic prover (i.e., with small prover communication complexity) can reveal, say, the first $\log n$ bits of the witness $w$ to the statement $x$ proved, yet Theorem 4 states that such a protocol satisfies a notion of zero-knowledge. But if we leak something specific about the witness, how can we expect the protocol to be "zero-knowledge"? The key point here is that (as shown in Theorem 4), for random statements $x$, the

\footnotetext{
${ }^{1}$ More specifically, the notion of "ultra-weak zero-knowledge" of 12 considers both
} of these relaxations, but relaxes the notion even further. 
information revealed about the witness can actually be efficiently generated. In other words, the whole process where the prover first picks the statement (at random), and then provides the proof, is zero-knowledge.

Despite the simplicity of the proof of Theorem 4, it has many (in our eyes) intriguing corollaries. The first one is that the classic two-round graph nonisomorphism protocol of [18] (which is only known to be "honest-verifier" zeroknowledge) is distributional $(t, \epsilon)$-zero-knowledge for every polynomial $t$ and every inverse polynomial $\epsilon 2$ In fact, by the complete problem for SZK [29], we can show that every language in SZK has a 2-round interactive proof that is distributional $(t, \epsilon)$-zero-knowledge for every polynomial $t$ and every inverse polynomial $\epsilon$.

Theorem 5 (Informally stated). For every language $L \in S Z K$ and every polynomial $p$, there exists a 2-round interactive proof $(P, V)$ for $L$ with completeness $1-\operatorname{negl}(\cdot)$ and soundness error $\frac{1}{p(\cdot)}$, and is distributional $(t, \epsilon)$-zeroknowledge for every polynomial $t$ and every inverse polynomial $\epsilon$.

We proceed to outline two other applications of Theorem 4.

Leakage Lemma of Gentry-Wichs. Roughly speaking, the "Leakage Lemma" of Gentry-Wichs [16] states that for every joint distribution $(X, \pi(X))$, where $|\pi(x)|=O(\log |x|)(\pi$ should be thought of as leakage on $X)$, and for every distribution $Y$ that is indistinguishable from $X$, there exists some leakage $\widetilde{\pi}$ such that the joint distributions $(X, \pi(X))$ and $(Y, \tilde{\pi}(Y))$ are indistinguishable. As we now argue, this lemma (and in fact, a stronger version of it) is a direct consequence of Theorem 4 .

In the language of zero-knowledge, let $X$ be a distribution over statements, and consider a one-message interactive proof where $\pi(x)$ denotes the distribution over the prover's message when the statement is $x$. By Theorem 4. this protocol is distributional zero-knowledge, and thus there exists an efficient simulator $S$ that can simulate the interaction (i.e, $(X, S(X))$ is indistinguishable from $(X, \pi(X)))$. By the indistinguishability of $Y$ and $X$ (and the efficiency of $S$ ), it directly follows that $(Y, S(Y))$ is indistinguishable from $(X, \pi(X))$. Thus we have found $\widetilde{\pi}=S$.

Let us note that our proof of the leakage lemma yields an even stronger statement - namely, we have found an efficient simulator $\tilde{\pi}$; such a version of the leakage lemma was recently established by Jetchev and Pietrzak 23. (As an independent contribution, our proof of Theorem 4 is actually significantly simpler than both the proof of [16] and [23.) Additionally, since our result on zero-knowledge applies also to interactive protocols, we directly also get an interactive version of the leakage lemma.

Dense Model Theorem. Roughly speaking, the Dense Model Theorem of 2830 states that if $X$ is indistinguishable from the uniform distribution over

\footnotetext{
${ }^{2}$ Recall that in the classic Graph Non-Isomorphism protocol the prover sends just a
} single bit and thus is very laconic. 
$n$-bits, $U_{n}$, and $R$ is $\delta$-dense 3 in $X$, then there exists a "model-distribution" $M$ that is (approximately) $\delta$-dense in $U_{n}$ such that $M$ is indistinguishable from $R$. Again, we show that this lemma is a direct consequence of Theorem 4. (Furthermore, our proof of Theorem 4 is arguably simpler and more modular than earlier proofs of the dense model theorem.)

Let us first translate the statement of the dense model theorem into the language of zero-knowledge. Let $X$ be a distribution over statements $x$, and consider some distribution $R$ that is $\delta$-dense in $X$, i.e., there exists a joint distribution $(X, B(X))$ with $\operatorname{Pr}[B(X)=1] \geq \delta$ such that $R=X \mid(B(X)=1)$. Define a one-bit proof where the prover sends the bit $B(x)$, where $x$ is the statement. By Theorem 4, there exists a simulator $S$ for this interactive proof; let $M=U_{n} \mid\left(S\left(U_{n}\right)=1\right)$. By the indistinguishability of the simulation, $(X, S(X))$ is indistinguishable from $(X, B(X))$, and thus by indistinguishability of $X$ and $U_{n},\left(U_{n}, S\left(U_{n}\right)\right)$ is indistinguishable from $(X, B(X))$. It follows that $M$ is (approximately) $\delta$-dense in $U_{n}$, and $M$ is indistinguishable from $R$.

\subsection{A Note on Our Non-Black-Box Simulation Technique}

The universal simulators in Theorem 3, 4, and 5] are indirectly obtained via the minimax theorem used in the proof of Theorem 3, and again we make non-blackbox usage of the verifier $V^{*}$. We remark that our non-black-box usage of $V^{*}$ is necessary (assuming standard complexity-theoretic assumptions): We show that black-box simulation techniques cannot be used to demonstrate distributional $(t, \epsilon)$ zero-knowledge for 2-round proof systems for languages that are hard-on-average.

Theorem 6 (Informally stated). Let $L$ be any language that is hard-onaverage for polynomial-size circuits, and let $(P, V)$ be any 2-round interactive proof (with completeness $2 / 3$ and soundness error $1 / 3$ ) for $L$. Then, there exists a polynomial $t$ such that for every $\epsilon(n)<1 / 12,(P, V)$ is not black-box distributional $(t, \epsilon)$-zero-knowledge

As as consequence we have that as long as SZK contains a language that is hardon-average, our non-black-box techniques are necessary (otherwise, Theorems 5 and 6 would contradict each other). As far as we know, the above yields the first example where a non-black-box simulation technique can be used to analyze "natural" protocols (e.g., the classic graph non-isomorphism protocol) that were not "tailored" for non-black-box simulation, but for which black-box simulation is not possible. This stands in sharp contrast to the non-black-box technique of Barak [2] and its follow-ups (see e.g., [26 25 27/3 11/5/106]), where non-black-box simulation is enabled by a very specific protocol design. This gives hope that non-black-box techniques can be used to analyze simple/practical protocols.

${ }^{3} R$ is said to be $\delta$-dense in $X$ if for every $r, \operatorname{Pr}[R=r] \leq(1 / \delta) \cdot \operatorname{Pr}[X=r]$; equivalently, $R$ is $\delta$-dense in $X$ if there exists a joint distribution $(X, B(X))$ with $\operatorname{Pr}[B(X)=1] \geq$ $\delta$ such that $R=X \mid(B(X)=1)$. 
Let us finally remark that in our non-black-box technique, we only need to make non-black-box use of the malicious verifier $V^{*}$ 's auxiliary input $z$ and its running-time $t$, but otherwise we may treat $V^{*}$ 's Turing machine as a blackbox. Although the non-black-box simulation technique of Barak [2] also makes non-black-box usage of $V^{*}$ 's Turing machine, it is not hard to see that also this technique can be modified to only make non-black-box usage of $z$ and $t$ (but not its Turing machine) - since the description of $V^{*}$ 's Turing machine is of constant length the non-black-box simulator can simply enumerate all possible Turing machines in the protocol of Barak.

\subsection{Our Techniques}

As mentioned, both Theorem 2 and 3 rely on the minimax theorem from game theory. Recall that the minimax theorem states that in any finite two-player zero-sum game, if for every distribution over the actions of Player 1, there exists some action for Player 2 that guarantees him an expected utility of $v$, then there exists some (universal) distribution of actions for Player 2 such that no matter what action Player 1 picks, Player 2 is still guaranteed an expected utility of $v$. For us, Player 1 will be choosing a distinguisher, and Player 2 will be choosing a simulator; roughly speaking, Player 2's utility will be "high" if the simulation is "good" for the distinguisher chosen by Player 1 . Now, by the weak zero-knowledge property, we are guaranteed that for every distinguisher chosen by Player 1, there exists some simulator for Player 2 that guarantees him a high utility. Thus intuitively, by the minimax theorem, Player 2 should have a simulator that yields him high utility with respect to any distinguisher.

There are two problems with this approach. First, to apply the minimax theorem, we require the existence of a good "distinguisher-dependent" simulator for every distribution over distinguishers. Secondly the minimax theorem only guarantees the existence of a distribution over simulators that works well against every distinguisher. We resolve both of these issues in quite different ways for Theorem 3 and Theorem 2 .

In the context of Theorem 3 , since we model both the simulator and distinguisher as non-uniform machines, we can use standard techniques to "derandomize" any distribution over simulators/distinguishers into a single simulator/distinguisher that gets some extra non-uniform advice: we simply approximate the original distribution by sufficiently many samples from it, and these samples can be provided to a single machine as non-uniform advice. (Such "derandomization" techniques originated in the proof of the hard-core lemma [22].)

In the context of Theorem 2 the situation is more difficult since we need both the distinguisher and the simulator to be uniform. In particular, we are only guaranteed the existence of a good distinguisher-dependent simulator for every uniform distinguisher and not necessarily for non-uniform ones. Here, we instead try to efficiently and uniformly find the "minimax" distribution over simulator strategies. If this can be done, then we do have a single uniform (and efficient) simulator algorithm. Towards this, we use a multiplicative weights algorithm, which can be used to approximately find the minimax strategies of two-player 
zero-sum games (e.g., see 14]). The multiplicative weights algorithm roughly works as follows. In the first round, Player 1 chooses the uniform distribution over the set of all $t(n)$-time Turing machines with description size $\leq \log n$ (note that any $t(n)$-time uniform distinguisher will be a member of this set for sufficiently large $n$ ), and then Player 2 chooses a "good simulator" that yields high payoff with respect to Player 1's distribution (note that since Player 1's distribution is uniformly and efficiently computable, we can view the process of sampling from it, and next running the sampled distinguisher, as a single uniform and efficient distinguisher, and thus we may rely on the weak zero-knowledge definition to conclude that a good simulator exists). In the next round, Player 1 updates its distribution using a multiplicative update rule that depends on Player 2's chosen simulator in the previous round; Player 2 again chooses a simulator that yields high payoff with respect to Player 1's new distribution, etc. By repeating this procedure for polynomially many rounds, Player 2 obtains a sequence of simulators such that the uniform distribution over the multiset of simulators yields high payoff no matter what distinguisher Player 1 chooses.

There are some issues that need to be resolved. In each round, we need to pick a simulator that works well against a (uniformly and efficiently computable) distribution over $t(n)$-time distinguishers. Although the running-time of the underlying distinguishers is bounded by $t(n)$, the time needed to sample from this distribution could be growing (exponentially) in each round, which in turn could potentially lead to an exponential growth in the running-time of the simulator. Thus after polynomially many rounds, it is no longer clear that the simulator or the distribution over distinguishers is polynomial-time 4 To deal with this issue, we rely on the "good" distinguisher-dependent simulator for a single universal distinguisher that receives as auxiliary input the code of the actual distinguisher it is running; we can then at each step approximate the distribution over distinguishers and feed this approximation as auxiliary input to the universal distinguisher.

Another important issue to deal with is the fact that to evaluate the "goodness" of a simulation w.r.t. to some distinguisher (i.e., to compute the utility function), we need to be able to sample true views of the malicious verifier in an interaction with the honest prover-but if we could do this, then we would already be done! Roughly speaking, we overcome this issue by showing that the goodness of a simulation w.r.t. a particular distinguisher $D$ can be approximated by using the distinguisher-dependent simulator $S_{D}$ for $D$.

We remark that in both of the above proofs, the reason that we work with a $(t, \epsilon)$-notion of zero-knowledge is that the running-time of the simulator we construct is polynomial in $t$ and $1 / \epsilon$.

\subsection{Related Work}

As mentioned above, the notion of weak zero-knowledge was first introduced by Dwork, Naor, Reingold and Stockmeyer [12. Dwork et al also considered non-

\footnotetext{
${ }^{4}$ A similar issue appeared in a recent paper by us in the context of forecast testing 9], where we used a related, but different, technique to overcome it.
} 
uniform versions and distributional versions of zero-knowledge; distributional versions of zero-knowledge were first considered by Goldreich [17] in a uniform setting (called uniform zero-knowledge).

The minimax theorem from game-theory has been applied in various contexts in complexity theory (e.g., 22 4 283031]) and more recently also in cryptography (e.g., [2813816 3123]). The proof of Theorem4 4 is related to the approaches taken in these previous works, and most closely related to the approach taken in [30]. However, as far as we know, none of the earlier results have applied the minimax theorem in the context of zero-knowledge. Nevertheless, as we mentioned above, our Theorem 4 implies some of these earlier results (and shows that they can be understood in the language of zero-knowledge).

In a recent paper [31, Vadhan and Zheng proved a uniform minimax theorem, but our usage of the multiplicative weights algorithm cannot be simplified by using their uniform minimax theorem. One reason is that in our setting, the payoff (utility) function of the zero-sum game cannot be efficiently computed, and thus we have to approximate it. The uniform minimax theorem of 31] does not handle the usage of an approximate payoff function (their theorem does allow the usage of approximate KL projections in the algorithm, but from what we can see, this is not sufficient for handling our approximate payoff function).

\subsection{Overview}

In Section 2, we show that weak zero-knowledge is not equivalent to zeroknowledge (Theorem 1 above). In Section [3, we show that weak and strong $(t, \epsilon)$-zero-knowledge are equivalent (Theorem 2 above). In Section 4 we show that super-weak and strong distributional $(t, \epsilon)$-zero-knowledge are equivalent (Theorem 3 above), and interactive proofs with a laconic prover are distributional zero-knowledge (Theorem 4 above), and we also describe applications of this result. In the full version of this paper, we separate the notion of super-weak and weak $(t, \epsilon)$-zero-knowledge.

\section{Separation of Weak and Strong Zero-Knowledge}

Given a prover $P$, a verifier $V^{*}$, and $x, z \in\{0,1\}^{*}$, let $O u t_{V^{*}}\left[P(x) \leftrightarrow V^{*}(x, z)\right]$ denote the output of $V^{*}(x, z)$ after interacting with $P(x)$. We now state the definition of zero-knowledge for convenient reference.

Definition 1 (zero-knowledge). Let $(P, V)$ be an interactive proof system for a language $L$. We say that $(P, V)$ is zero-knowledge if for every PPT adversary $V^{*}$, there exists a PPT simulator $S$ such that for every PPT distinguisher $D$, there exists a negligible function $\nu(\cdot)$ such that for every $n \in \mathbb{N}, x \in L \cap\{0,1\}^{n}$, and $z \in\{0,1\}^{*}$, we have

$$
\mid \operatorname{Pr}\left[D\left(x, z, \text { Out }_{V^{*}}\left[P(x) \leftrightarrow V^{*}(x, z)\right]\right)=1\right]-\operatorname{Pr}[D(x, z, S(x, z))=1] \mid \leq \nu(n) .
$$


Remark 1. If $L$ is a language in NP with witness relation $R_{L}$, we usually require the prover $P$ to be efficient, but on common input $x$, we also give any witness $y \in R_{L}(x)$ to the prover $P$. We refer to such a notion as efficient prover zeroknowledge. More formally, in the definition of zero-knowledge above, we would change " $x \in L \cap\{0,1\}^{n}$, and $z \in\{0,1\}^{*}$ " to " $x \in L \cap\{0,1\}$ ",$y \in R_{L}(x)$, and $z \in\{0,1\}^{*}$, and we would change $P(x)$ to $P(x, y)$ and require $P$ to be efficient. All subsequent definitions can be extended to an efficient prover setting in an obvious way.

One can relax the definition of zero-knowledge by switching the order of the quantifiers $\exists S$ and $\forall D$ so that the simulator $S$ can depend on the distinguisher $D$. We call the relaxed definition weak zero-knowledge (following [12]).

Definition 2 (weak zero-knowledge). Let $(P, V)$ be an interactive proof system for a language $L$. We say that $(P, V)$ is weak zero-knowledge if for every PPT adversary $V^{*}$ and every PPT distinguisher $D$, there exists a PPT simulator $S$ and a negligible function $\nu(\cdot)$ such that for every $n \in \mathbb{N}, x \in L \cap\{0,1\}^{n}$, and $z \in\{0,1\}^{*}$, we have

$$
\mid \operatorname{Pr}\left[D\left(x, z, \text { Out }_{V^{*}}\left[P(x) \leftrightarrow V^{*}(x, z)\right]\right)=1\right]-\operatorname{Pr}[D(x, z, S(x, z))=1] \mid \leq \nu(n) .
$$

We now show that weak zero-knowledge is not equivalent to zero-knowledge if we assume the existence of two-round "timed" commitment schemes and "timed" worst-case weak one-way permutations satisfying certain properties. More precisely, we assume that there exists a polynomial $p(\cdot)$ such that for sufficiently large $n \in \mathbb{N}$, the following hold:

- There exists a collection of two-round "timed" commitment schemes $\left\{\operatorname{Com}_{i}\right\}_{i \in[\ell]}$, where $\ell=\log ^{2} n$, such that $\operatorname{Com}_{i}$ is hard to break in $p(n)^{i-1}$ steps, but can always be broken in $p(n)^{i}$ steps to obtain the committed value (e.g., one can get such timed commitment schemes from a timed commitment scheme in [7]).

- There exists a collection of "timed" worst-case weak one-way permutations $\left\{f_{i}\right\}_{i \in[\ell]}$, where $\ell=\log ^{2} n$, such that $f_{i}$ is somewhat hard to invert in $p(n)^{i+1}$ steps in the worst case (i.e., an adversary running in $p(n)^{i+1}$ steps will fail to invert some instance $f_{i}\left(x^{\prime}\right)$ with probability at least $\left.1 / \operatorname{poly}(n)\right)$, but can always be inverted in $p(n)^{i+2}$ steps.

Theorem 7. Assume the existence of two-round "timed" commitment schemes and "timed" worst-case weak one-way permutations as described above. Then, there exists an interactive proof system $(P, V)$ for an $N P$ language $L$ such that $(P, V)$ is weak zero-knowledge but not zero-knowledge.

Proof (Proof sketch). The proof roughly works as follows. Let $L$ be the trivial NP language $\{0,1\}^{*}$ with witness relation $R_{L}(x)=\left\{\left(f_{1}^{-1}(x), \ldots, f_{\log ^{2}|x|}^{-1}(x)\right)\right\}$.

Let $(P(x, y), V(x))$ be the following interactive proof, where $x \in\{0,1\}^{*}, n=$ $|x|, \ell=\log ^{2} n$, and $y=\left(f_{1}^{-1}(x), \ldots, f_{\ell}^{-1}(x)\right)$ :

1. The verifier $V$ generates and sends $\rho_{i}$ for $i=1, \ldots, \ell$ to the prover, where $\rho_{i}$ is the first message of an execution of $\operatorname{Com}_{i}$. 
2. The prover $P$ sends $\operatorname{Com}_{i}\left(f_{i}^{-1}(x), \rho_{i}\right)$ for $i=1, \ldots, \ell$ to the verifier, where $\operatorname{Com}_{i}(v, r)$ denotes the commitment of $v$ using $\operatorname{Com}_{i}$ with first message $r$.

3. The verifier $V$ accepts (i.e., outputs 1 ).

To see that $(P, V)$ is weak zero-knowledge, consider any PPT verifier $V^{*}$ and any PPT distinguisher $D$, and let $T(n)$ be a polynomial that bounds the combined running time of $V^{*}$ and $D$. Then, a simulator $S$ can compute the smallest positive integer $j$ such that $p(n)^{j-1}>T(n)$, and then break $f_{1}^{-1}(x), \ldots, f_{j-1}^{-1}(x)$ in polynomial time. Then, the simulator $S$ can simulate the protocol except that for $i=j, \ldots, \ell$, the simulator $S$ sends $\operatorname{Com}_{i}\left(0^{n}, \rho_{i}\right)$ to $V^{*}$ since $S$ does not know $f_{i}^{-1}(x)$. By the hiding property of $\operatorname{Com}_{j}, \ldots, \operatorname{Com}_{\ell}$, the distinguisher $D$ cannot distinguish between the output of the verifier $V^{*}$ (in a true interaction with $P$ ) and the output of the simulator $S$, since $D$ and $V^{*}$ (combined) cannot break any of the commitment schemes $\operatorname{Com}_{j}, \ldots, \operatorname{Com}_{\ell}$ (since $D$ and $V^{*}$ do not run long enough).

Intuitively, $(P, V)$ is not zero-knowledge because the existence of a (universal) simulator $S$ would allow us to invert a worst-case weak one-way permutation $f_{j}$ with overwhelming probability and in less time than what is specified in our hardness assumption for $f_{j}$. To see this, consider a PPT distinguisher $D$ that, given $x$ and a view of $V$, runs longer than $S$ and breaks a commitment $\operatorname{Com}_{j}\left(w_{j}, \rho_{j}^{\prime}\right)$ from the view of $V$ such that the time needed to break $f_{j}$ is much longer than the running time of the simulator $S$, and then verifies whether or not $f\left(w_{j}\right)=x$. The fact that the simulator $S$ works for the distinguisher $D$ will ensure that with overwhelming probability, the output of $S(x)$ will contain a commitment $\operatorname{Com}_{j}\left(w_{j}, \rho_{j}^{\prime}\right)$ of some $w_{j}$ such that $f_{j}\left(w_{j}\right)=x$. Thus, we can now construct an adversary $A$ that inverts $f_{j}\left(w_{j}\right)$ with overwhelming probability by running the simulator $S$ on input $f_{j}\left(w_{j}\right)$ and breaking the commitment $\operatorname{Com}_{j}\left(w_{j}, \rho_{j}^{\prime}\right)$ in the output of $S$. Since breaking $f_{j}$ takes longer time than running the simulator $S$ and breaking the commitment $\operatorname{Com}_{j}\left(w_{j}, \rho_{j}^{\prime}\right)$, the adversary $A$ contradicts our hardness assumption for $f_{j}$.

See the full version of this paper for the full proof of Theorem 7 .

\section{From Weak to Strong $(t, \epsilon)$-Zero-Knowledge}

From Theorem 7, we know that zero-knowledge and weak zero-knowledge are not equivalent. Thus, we now consider relaxed notions of zero-knowledge. We first consider a concrete security variant of the notion of zero-knowledge.

Definition 3 ( $(t, \epsilon)$-zero-knowledge). Let $(P, V)$ be an interactive proof system for a language $L$. We say that $(P, V)$ is $(t, \epsilon)$-zero-knowledge if for every PPT adversary $V^{*}$, there exists a PPT simulator $S$ such that for every $t$-time distinguisher $D$, there exists an $n_{0} \in \mathbb{N}$ such that for every $n \geq n_{0}, x \in L \cap\{0,1\}^{n}$, and $z \in\{0,1\}^{*}$, we have

$$
\mid \operatorname{Pr}\left[D\left(x, z, \text { Out }_{V^{*}}\left[P(x) \leftrightarrow V^{*}(x, z)\right]\right)=1\right]-\operatorname{Pr}[D(x, z, S(x, z))=1] \mid \leq \epsilon(n) .
$$


Similar to before, we can relax the definition of zero-knowledge by switching the order of the quantifiers $\exists S$ and $\forall D$ so that the simulator $S$ can depend on the distinguisher $D$. We call the relaxed definition weak $(t, \epsilon)$-zero-knowledge.

Definition 4 (weak $(t, \epsilon)$-zero-knowledge). Let $(P, V)$ be an interactive proof system for a language $L$. We say that $(P, V)$ is weak $(t, \epsilon)$-zero-knowledge if for every PPT adversary $V^{*}$ and every t-time distinguisher $D$, there exists a PPT simulator $S$ and an $n_{0} \in \mathbb{N}$ such that for every $n \geq n_{0}, x \in L \cap\{0,1\}^{n}$, and $z \in\{0,1\}^{*}$, we have

$$
\mid \operatorname{Pr}\left[D\left(x, z, \text { Out }_{V^{*}}\left[P(x) \leftrightarrow V^{*}(x, z)\right]\right)=1\right]-\operatorname{Pr}[D(x, z, S(x, z))=1] \mid \leq \epsilon(n) .
$$

Note that if $(P, V)$ is $(t, \epsilon)$-zero-knowledge (resp. weak $(t, \epsilon)$-zero-knowledge) for some super polynomial function $t$ and some negligible function $\epsilon$, then $(P, V)$ is zero-knowledge (resp. weak zero-knowledge) in the classic sense. We now show that $(t, \epsilon)$-zero-knowledge and weak $(t, \epsilon)$-zero-knowledge are equivalent if we consider all polynomials $t$ and inverse polynomials $\epsilon$.

Theorem 8. Let $(P, V)$ be an interactive proof system for a language $L$. Then, $(P, V)$ is weak $(t, \epsilon)$-zero-knowledge for every polynomial $t$ and inverse polynomial $\epsilon$ if and only if $(P, V)$ is $\left(t^{\prime}, \epsilon^{\prime}\right)$-zero-knowledge for every polynomial $t^{\prime}$ and inverse polynomial $\epsilon^{\prime}$.

Proof. The "if" direction clearly holds by definition. We will now prove the "only if" direction. Suppose $(P, V)$ is weak $(t, \epsilon)$-zero-knowledge for every polynomial $t$ and inverse polynomial $\epsilon$. Let $t^{\prime}$ be any polynomial, and let $\epsilon^{\prime}$ be any inverse polynomial.

Let $V^{*}$ be any PPT adversary, and let $T_{V^{*}}(\cdot)$ be any polynomial that bounds the running time of $V^{*}$. It is not hard to see that without loss of generality, we can assume that the auxiliary input $z \in\{0,1\}^{*}$ in the definition of $\left(t^{\prime}, \epsilon^{\prime}\right)$-zeroknowledge is exactly $C \cdot\left(T_{V^{*}}(n)+t^{\prime}(n)\right)$ bits long, where $C$ is some constant $\geq 15$ Furthermore, it is easy to see that without loss of generality, we can also remove the absolute value $|\cdot|$ and change $\epsilon^{\prime}(n)$ to $O\left(\epsilon^{\prime}(n)\right)$. Thus, it suffices to construct a PPT simulator $S$ such that for every $t^{\prime}$-time distinguisher $D$, there exists an $n_{0} \in \mathbb{N}$ such that for every $n \geq n_{0}, x \in L \cap\{0,1\}^{n}$, and $z \in\{0,1\}^{*}$ with $|z|=C \cdot\left(T_{V^{*}}(n)+t^{\prime}(n)\right)$, we have

$$
\operatorname{Pr}\left[D\left(x, z, O u t_{V^{*}}\left[P(x) \leftrightarrow V^{*}(x, z)\right]\right)=1\right]-\operatorname{Pr}[D(x, z, S(x, z))=1] \leq O\left(\epsilon^{\prime}(n)\right) .
$$

We will now construct the required PPT simulator $S$ for $V^{*}$.

High-level Description of the Simulator $S$ : We first give a high-level description of the simulator $S$. The simulator $S$ uses the multiplicative weights algorithm described in [14]. The simulator $S$, on input $(x, z)$ with $n:=|x|$, first runs a multiplicative weights algorithm to find a "good set" of simulator

\footnotetext{
${ }^{5}$ This follows from standard padding techniques and the fact that the adversary $V^{*}$ and the distinguisher $D$ cannot read any of the bits after the first $T_{V^{*}}(n)+t^{\prime}(n)$ bits of $z$.
} 
machines $\left\{S_{1}, \ldots, S_{L}\right\}$; then, the simulator $S$ randomly and uniformly chooses one of the simulator machines in $\left\{S_{1}, \ldots, S_{L}\right\}$ to perform the simulation, i.e., $S$ runs the chosen simulator machine on input $(x, z)$ and outputs whatever the simulator machine outputs.

Before we describe the multiplicative weights algorithm run by the simulator $S$, let us introduce some notation. Given a simulator $S^{\prime}$ and a distinguisher $D^{\prime}$, let the "payoff" of $S^{\prime}$ (with respect to $D^{\prime}$ ) be

$\mu\left(S^{\prime}, D^{\prime}\right):=\operatorname{Pr}\left[D^{\prime}\left(x, z, S^{\prime}(x, z)\right)=1\right]-\operatorname{Pr}\left[D^{\prime}\left(x, z, O u t_{V^{*}}\left[P(x) \leftrightarrow V^{*}(x, z)\right]\right)=1\right]$.

Given a simulator $S^{\prime}$ and a distribution $\mathcal{D}^{(i)}$ over distinguishers, let

$$
\mu\left(S^{\prime}, \mathcal{D}^{(i)}\right):=\mathbb{E}_{D^{\prime} \sim \mathcal{D}^{(i)}}\left[\mu\left(S^{\prime}, D^{\prime}\right)\right]=\sum_{D^{\prime} \in \operatorname{Supp}\left(\mathcal{D}^{(i)}\right)} \mathcal{D}^{(i)}\left(D^{\prime}\right) \cdot \mu\left(S^{\prime}, D^{\prime}\right) .
$$

We note that we want to design the simulator $S$ so that for every $t^{\prime}$-time distinguisher $D$, we have $\mu(S, D) \geq-O\left(\epsilon^{\prime}(n)\right)$.

Let $D_{1}, D_{2}, D_{3}, \ldots$ be an enumeration of the set of all (uniform) distinguishers, and let $D_{1}^{\prime}, D_{2}^{\prime}, D_{3}^{\prime}, \ldots$ be the corresponding sequence where $D_{j}^{\prime}$ is the same as $D_{j}$ except that after $t^{\prime}(n)$ steps, $D_{j}^{\prime}$ stops and outputs 0 . We note that each fixed $t^{\prime}$-time distinguisher $D$ will eventually appear in the set $\left\{D_{1}^{\prime}, \ldots, D_{n}^{\prime}\right\}$ as $n$ gets larger.

We now describe the multiplicative weights algorithm run by $S$. In the multiplicative weights algorithm, $S$ simulates $L$ rounds (repetitions) of a zero-sum game between a "simulator player" Sim and a "distinguisher player" Adv, where the payoff function for Sim is the function $\mu(\cdot, \cdot)$ defined above. In each round $i$, Adv chooses a mixed strategy (i.e., a distribution) $\mathcal{D}^{(i)}$ over its set of pure strategies $\left\{D_{1}^{\prime}, \ldots, D_{n}^{\prime}\right\}$ (a set of distinguishers), and then Sim chooses a simulator machine $S_{i}:=S_{i}\left(\mathcal{D}^{(i)}\right)$ that hopefully "does well" against Adv's mixed strategy $\mathcal{D}^{(i)}$, i.e., Sim's (expected) payoff $\mu\left(S_{i}, \mathcal{D}^{(i)}\right)$ is high.

In the first round, Adv chooses the uniform distribution $\mathcal{D}^{(1)}$ over $\left\{D_{1}^{\prime}, \ldots, D_{n}^{\prime}\right\}$. After each round $i$, Adv updates its mixed strategy to get $\mathcal{D}^{(i+1)}$ in a manner similar to the multiplicative weights algorithm described in [14], which involves the payoff function $\mu$. However, Adv cannot compute $\mu$ efficiently, since $\mu$ involves the prover $P$, which may be inefficient (or has a witness $y$ that Adv does not have). Thus, Adv uses an approximation $\widehat{\mu}$ of the payoff function $\mu$. In particular, given a distinguisher $D^{\prime}$, Adv can approximate $\mu\left(S_{i}, D^{\prime}\right)$ by approximating $O u t_{V^{*}}[P(x) \leftrightarrow$ $\left.V^{*}(x, z)\right]$ with the output of a simulator $S_{D^{\prime}}$ that is good w.r.t. the distinguisher $D^{\prime}$; the existence of such a simulator is guaranteed by the weak zero-knowledge property of $(P, V)$. There are still some issues: Adv might not be able to find $S_{D^{\prime}}$ efficiently and uniformly, and $S_{D^{\prime}}$ only works well for sufficiently large $n$. We resolve these issues by using a "universal" distinguisher that essentially takes a description of a distinguisher $D^{\prime}$ as auxiliary input and runs $D^{\prime}$, and we use a simulator that is good w.r.t. this universal distinguisher.

Using an analysis similar to that in [14, we will show that if Sim manages to choose a simulator machine $S_{i}$ that does well against Adv's mixed strategy $\mathcal{D}^{(i)}$ in every round $i \in[L]$, then the uniform mixed strategy over the set 
$\left\{S_{1}, \ldots, S_{L}\right\}$ of chosen simulator machines does well against all the distinguishers in $\left\{D_{1}^{\prime}, \ldots, D_{n}^{\prime}\right\}$. To choose a simulator machine $S_{i}$ that does well against Adv's mixed strategy $\mathcal{D}^{(i)}$, Sim makes use of the weak zero-knowledge property of $(P, V)$, which guarantees that for every distinguisher $D$, there exists a simulator $S_{D}$ that does well against $D$. However, there are some complications: $(1) \mathcal{D}^{(i)}$ is a mixture of distinguishers, not a single distinguisher; (2) Sim might not be able to efficiently and uniformly find the distinguisher-dependent simulator; and (3) even if Sim can efficiently and uniformly find the distinguisher-dependent simulator, the simulator depends on the mixed strategy $\mathcal{D}^{(i)}$, and the time needed to sample from $\mathcal{D}^{(i)}$ could be growing (exponentially) in each round, which in turn can potentially lead to an exponential growth in the running time of the distinguisher-dependent simulator as more rounds are performed.

Sim overcomes these problems by (also) using a "universal" distinguisher $D_{U}$ that takes the weights (i.e., probability masses) of a distribution $\mathcal{D}$ over $\left\{D_{1}^{\prime}, \ldots, D_{n}^{\prime}\right\}$ as auxiliary input, samples a distinguisher from the distribution $\mathcal{D}$, and then runs the sampled distinguisher. Let $S_{D_{U}}$ be the simulator that is good w.r.t. $D_{U}$; again, the existence of such a simulator is guaranteed by the weak zero-knowledge property of $(P, V)$. Sim chooses $S_{i}$ to be the simulator machine that runs $S_{D_{U}}$ with the weights of the distribution $\mathcal{D}^{(i)}$ provided as auxiliary input. We now give a formal description of the simulator $S$.

The Simulator $S$ : Let $D_{1}, D_{2}, D_{3}, \ldots$ be an enumeration of the set of all (uniform) distinguishers, and let $D_{1}^{\prime}, D_{2}^{\prime}, D_{3}^{\prime}, \ldots$ be the corresponding sequence where $D_{j}^{\prime}$ is the same as $D_{j}$ except that after $t^{\prime}(n)$ steps, $D_{j}^{\prime}$ stops and outputs 0 .

The simulator $S$, on input $(x, z)$ with $n:=|x|$, proceeds as follows:

1. Let $T_{D_{U}}(n)=O\left(\left(T_{V^{*}}(n)+t^{\prime}(n)+n\right)^{2}\right)$.

Given a distribution $\mathcal{D}$ over $\left\{D_{1}^{\prime}, \ldots, D_{n}^{\prime}\right\}$, let $\boldsymbol{p}_{\mathcal{D}}$ denote the vector of weights (i.e., probability masses) representing $\mathcal{D}$, i.e., $\boldsymbol{p}_{\mathcal{D}}=\left(\mathcal{D}\left(D_{1}^{\prime}\right), \ldots, \mathcal{D}\left(D_{n}^{\prime}\right)\right)$.

Let $D_{U}$ be a "universal" distinguisher that, on input $\left(x, z^{\prime}, v\right)$, first parses $z^{\prime}$ as $z^{\prime}=z \| \boldsymbol{p}_{\mathcal{D}}$, where $\boldsymbol{p}_{\mathcal{D}}$ is a vector the weights representing some distribution $\mathcal{D}$ over $\left\{D_{1}^{\prime}, \ldots, D_{n}^{\prime}\right\}$; then, $D_{U}$ samples a distinguisher $D_{j}^{\prime}$ from the distribution $\mathcal{D}$, and then runs $D_{j}^{\prime}$ on input $(x, z, v)$, but $D_{U}$ always stops after $T_{D_{U}}(n)$ steps regardless of whether or not $D_{j}^{\prime}$ finishes running.

Let $S_{D_{U}}$ be the PPT simulator for $D_{U}$ that is guaranteed by the weak $\left(T_{D_{U}}, \epsilon^{\prime}\right)$-zero-knowledge property of $(P, V)$.

2. Let $L=\Theta\left(\frac{\log n}{\epsilon^{\prime}(n)^{2}}\right)$ and $\beta=\frac{1}{1+\sqrt{(2 \ln n) / L}}$. ( $L$ is the number of rounds we will run the multiplicative weights algorithm for, and $\beta$ is used in the multiplicative update rule.)

3. Multiplicative weights algorithm:

Let $\mathcal{D}^{(1)}$ be the uniform distribution over $\left\{D_{1}^{\prime}, \ldots, D_{n}^{\prime}\right\}$. (The probability mass $\mathcal{D}^{(1)}\left(D_{j}^{\prime}\right)$ for $D_{j}^{\prime}$ can be thought of as the "weight" for $D_{j}^{\prime}$.)

For $i=1, \ldots, L$ do:

(a) Choosing a simulator machine $S_{i}$ that does well against $\mathcal{D}^{(i)}$ :

Let $S_{i}$ be a simulator machine that, on input $(x, z)$, outputs $S_{D_{U}}\left(x, z \| \boldsymbol{p}_{\mathcal{D}^{(i)}}\right)$.

(b) Weight update: 
Compute the distribution $\mathcal{D}^{(i+1)}$ from $\mathcal{D}^{(i)}$ by letting

$$
\mathcal{D}^{(i+1)}\left(D_{j}^{\prime}\right) \sim \beta^{\widehat{\mu}\left(S_{i}, D_{j}^{\prime}\right)} \cdot \mathcal{D}^{(i)}\left(D_{j}^{\prime}\right)
$$

for every $D_{j}^{\prime} \in\left\{D_{1}^{\prime}, \ldots, D_{n}^{\prime}\right\}$ (and renormalizing), where

$$
\widehat{\mu}\left(S_{i}, D_{j}^{\prime}\right):=\operatorname{freq}_{k}\left[D_{j}^{\prime}\left(x, z, S_{i}(x, z)\right)\right]-\operatorname{freq}_{k}\left[D_{j}^{\prime}\left(x, z, S_{D_{U}}\left(x, z \| \boldsymbol{p}_{D_{j}^{\prime}}\right)\right)\right],
$$

where $\operatorname{freq}_{k}\left[D_{j}^{\prime}\left(x, z, S_{i}(x, z)\right)\right]$ and $\operatorname{freq}_{k}\left[D_{j}^{\prime}\left(x, z, S_{D_{U}}\left(x, z \| \boldsymbol{p}_{D_{j}^{\prime}}\right)\right)\right]$ are approximations of $\operatorname{Pr}\left[D_{j}^{\prime}\left(x, z, S_{i}(x, z)\right)=1\right]$ and $\operatorname{Pr}\left[D_{j}^{\prime}\left(x, z, S_{D_{U}}\left(x, z \| \boldsymbol{p}_{D_{j}^{\prime}}\right)\right)\right.$ $=1$ ] by taking $k:=\Theta\left(\frac{\log \left(n L / \epsilon^{\prime}(n)\right)}{\epsilon^{\prime}(n)^{2}}\right)$ samples, respectively, and computing the relative frequency in which 1 is outputted.

The function $\widehat{\mu}$ should be viewed as being an approximation of the payoff function $\mu$.

\section{End for}

4. Choose $S_{i} \in\left\{S_{1}, \ldots, S_{L}\right\}$ uniformly at random.

5. Run the simulator $S_{i}$ on input $(x, z)$ and output $S_{i}(x, z)$.

We now continue with the formal proof. It can be easily verified that $S$ runs in time $\operatorname{poly}\left(n, t^{\prime}(n), \frac{1}{\epsilon^{\prime}(n)}\right)$. Let $D$ be any distinguisher whose running time is bounded by $t^{\prime}(n)$. Fix an integer $n$ that is sufficiently large so that the distinguisher $D$ appears in $\left\{D_{1}, \ldots, D_{n}\right\}$ and $S_{D_{U}}$ works for the distinguisher $D_{U}$ on input size $n$ for $x$. We note that the distinguisher $D$ also appears in $\left\{D_{1}^{\prime}, \ldots, D_{n}^{\prime}\right\}$, since the running time of $D$ is bounded by $t^{\prime}(n)$. Fix $x \in L \cap\{0,1\}^{n}$ and $z \in\{0,1\}^{*}$ with $|z|=C \cdot\left(T_{V^{*}}(n)+t^{\prime}(n)\right)$. To prove the theorem, it suffices to show that

$$
\mu(S, D) \geq-O\left(\epsilon^{\prime}(n)\right) .
$$

To show this, we will proceed as follows: (1) We first show that if, in every round $i$ the chosen simulator $S_{i}$ does well against the distribution $\mathcal{D}^{(i)}$ with respect to our approximation $\widehat{\mu}$ of $\mu$, then the simulator $S$ does well against $D$ with respect to $\widehat{\mu}$; this is the first lemma below; (2) We then show that the first lemma holds even if we replace $\widehat{\mu}$ with $\mu$; this is the second lemma below; (3) Finally, we show that in each round $i$, the chosen simulator $S_{i}$ indeed does well against the distribution $\mathcal{D}^{(i)}$ with respect to $\mu$.

We now proceed with the proof. For $i=1, \ldots, L$, let

$$
\widehat{\mu}\left(S_{i}, \mathcal{D}^{(i)}\right):=\mathbb{E}_{D^{\prime} \sim \mathcal{D}^{(i)}}\left[\widehat{\mu}\left(S_{i}, D^{\prime}\right)\right]=\sum_{k=1}^{n} \mathcal{D}^{(i)}\left(D_{k}^{\prime}\right) \cdot \widehat{\mu}\left(S_{i}, D_{k}^{\prime}\right) .
$$

One should view $\widehat{\mu}\left(S_{i}, \mathcal{D}^{(i)}\right)$ as an approximation of $\mu\left(S_{i}, \mathcal{D}^{(i)}\right)$.

Lemma 1. For every distinguisher $D_{j}^{\prime} \in\left\{D_{1}^{\prime}, \ldots, D_{n}^{\prime}\right\}$, if we run the simulator $S(x, z)$, then (with probability 1$) S(x, z)$ generates $\mathcal{D}^{(1)}, \ldots, \mathcal{D}^{(L)}$ and $S_{1}, \ldots, S_{L}$ such that

$$
\frac{1}{L} \sum_{i=1}^{L} \widehat{\mu}\left(S_{i}, D_{j}^{\prime}\right) \geq \frac{1}{L} \sum_{i=1}^{L} \widehat{\mu}\left(S_{i}, \mathcal{D}^{(i)}\right)-O\left(\epsilon^{\prime}(n)\right)
$$


The proof of Lemma 1 is essentially the same as a lemma found in [9], whose proof is very similar to the analysis of the multiplicative weights algorithm found in [14. In [14, the multiplicative weights algorithm updates the weights of $\mathcal{D}^{(i)}$ using the exact value of $\mu\left(S_{i}, D_{j}^{\prime}\right)$; here, we only have an approximation $\widehat{\mu}\left(S_{i}, D_{j}^{\prime}\right)$ of $\mu\left(S_{i}, D_{j}^{\prime}\right)$, but with minor changes, the analysis in [14] can still be used to show Lemma 1]. We provide a proof of Lemma 1 in the full version of this paper.

We now show that we can essentially replace the $\widehat{\mu}$ in Lemma 1 with $\mu$.

Lemma 2. For every $D^{\prime} \in\left\{D_{1}^{\prime}, \ldots, D_{n}^{\prime}\right\}$, if we run the simulator $S(x, z)$, then with probability $1-O\left(\epsilon^{\prime}(n)\right)$ over the random coins of $S, S(x, z)$ generates $\mathcal{D}^{(1)}, \ldots, \mathcal{D}^{(L)}$ and $S_{1}, \ldots, S_{L}$ such that

$$
\frac{1}{L} \sum_{i=1}^{L} \mu\left(S_{i}, D^{\prime}\right) \geq \frac{1}{L} \sum_{i=1}^{L} \mu\left(S_{i}, \mathcal{D}^{(i)}\right)-O\left(\epsilon^{\prime}(n)\right) .
$$

The proof of Lemma 2 roughly works as follows. We take Lemma 1 and show that each time we approximate $\mu$ via $\widehat{\mu}$, the approximation is good with high probability; this follows from Chernoff bounds and the fact that $S_{D_{U}}$ is a simulator for $V^{*}$ that is good with respect to the "universal" distinguisher $D_{U}$. Lemma 2 then follows from the union bound. See the full version of this paper for the proof of Lemma 2

To complete the proof of Theorem 8 , we will now show that $\mu(S, D) \geq$ $-O\left(\epsilon^{\prime}(n)\right)$. We first show that for every $i \in[L]$, we always have $\mu\left(S_{i}, \mathcal{D}^{(i)}\right) \geq$ $-O\left(\epsilon^{\prime}(n)\right)$. Fix $i \in[L]$. Now, we observe that

$$
\begin{aligned}
& \mu\left(S_{i}, \mathcal{D}^{(i)}\right) \\
= & \sum_{j=1}^{n} \mathcal{D}^{(i)}\left(D_{j}^{\prime}\right) \cdot\left(\operatorname{Pr}\left[D_{j}^{\prime}\left(x, z, S_{i}(x, z)\right)=1\right]-\operatorname{Pr}\left[D_{j}^{\prime}\left(x, z, O u t_{V^{*}}\left[P(x) \leftrightarrow V^{*}(x, z)\right]\right)=1\right]\right) \\
= & \operatorname{Pr}\left[D_{U}\left(x, z \| \boldsymbol{p}_{\mathcal{D}^{(i)}}, S_{i}(x, z)\right)=1\right]-\operatorname{Pr}\left[D_{U}\left(x, z \| \boldsymbol{p}_{\mathcal{D}^{(i)}}, \text { Out }_{V^{*}}\left[P(x) \leftrightarrow V^{*}(x, z)\right]\right)=1\right] \\
= & \operatorname{Pr}\left[D_{U}\left(x, z \| \boldsymbol{p}_{\mathcal{D}^{(i)}}, S_{D_{U}}\left(x, z \| \boldsymbol{p}_{\mathcal{D}^{(i)}}\right)\right)=1\right] \\
& -\operatorname{Pr}\left[D_{U}\left(x, z \| \boldsymbol{p}_{\mathcal{D}^{(i)}}, O u t_{V^{*}}\left[P(x) \leftrightarrow V^{*}\left(x, z \| \boldsymbol{p}_{\mathcal{D}^{(i)}}\right)\right]\right)=1\right] \\
\geq & -\epsilon^{\prime}(n)
\end{aligned}
$$

where the second equality follows from the definition of $D_{U}$, the third equality follows from the definition of $S_{i}$ and the fact that $V^{*}(x, z)=V^{*}\left(x, z \| \boldsymbol{p}_{\mathcal{D}^{(i)}}\right)$ (since $|z| \geq T_{V^{*}}(n)$ ), and the last inequality follows from the fact that $S_{D_{U}}$ is a simulator for $D_{U}$ in the weak $\left(t^{\prime}, \epsilon^{\prime}\right)$-zero-knowledge property of $(P, V)$.

Now, combining Lemma 2 and (2), we have that with probability $1-O\left(\epsilon^{\prime}(n)\right)$ over the randomness of $S, S(x, z)$ generates $S_{1}, \ldots, S_{L}$ such that

$$
\frac{1}{L} \sum_{i=1}^{L} \mu\left(S_{i}, D\right) \geq-O\left(\epsilon^{\prime}(n)\right)
$$

Now, recall that after generating $S_{1}, \ldots, S_{L}$, the simulator $S(x, z)$ chooses a uniformly random $S_{i} \in\left\{S_{1}, \ldots, S_{L}\right\}$ and runs $S_{i}(x, z)$. Thus, conditional on 
$S(x, z)$ generating a particular sequence $S_{1}, \ldots, S_{L}$, we have $\mu(S, D)=\sum_{i=1}^{L} \frac{1}{L}$. $\mu\left(S_{i}, D\right)$. Combining this with (3) (which holds with probability $1-O\left(\epsilon^{\prime}(n)\right.$ ) over the randomness of $S)$, we get

$$
\mu(S, D) \geq-O\left(\epsilon^{\prime}(n)\right)-O\left(\epsilon^{\prime}(n)\right)=-O\left(\epsilon^{\prime}(n)\right),
$$

as required. This completes the proof of Theorem 8 .

\section{From Super-Weak to Strong Distributional $(T, t, \epsilon)$-Zero-Knowledge}

In this section we consider a "super-weak" notion of zero-knowledge, where not only do we allow the simulator to depend on the distinguisher, but also, we only require that the simulator manages to make the distinguisher output 1 with at least as high probability (minus some "small" gap) as the probability that the distinguisher outputs 1 when given a true view of $V^{*}$. That is, we only consider "one-sided" indistinguishability. (Such a notion was previously considered in [21.)

In the full version of this paper, we show that super-weak $(t, \epsilon)$-zero-knowledge is not equivalent to weak $(t, \epsilon)$-zero-knowledge. Thus, we here consider an alternative "distributional" notion of zero-knowledge (a la [17]) where indistinguishability of the simulation is only required for any distribution over statements (and auxiliary inputs), and the simulator as well as the distinguisher can depend on the distribution. Additionally, we here model both the distinguisher and the simulator as non-uniform algorithms (as opposed to uniform ones). (The combination of these variants was previously considered by [12]). For concreteness, we also add a parameter $T$ to the definition and require that the simulator is of size at most $T(n)$, and thus we also bound the size of the malicious verifier $V^{*}$ by $t(n)$.

Definition 5 (distributional $(T, t, \epsilon)$-zero-knowledge). Let $(P, V)$ be an interactive proof system for a language $L$. We say that $(P, V)$ is distributional $(T, t, \epsilon)$-zero-knowledge if for every $n \in \mathbb{N}$, every joint distribution $\left(X_{n}, Y_{n}, Z_{n}\right)$ over $\left(L \cap\{0,1\}^{n}\right) \times\{0,1\}^{*} \times\{0,1\}^{*}$, and every randomized $t(n)$-size adversary $V^{*}$, there exists a randomized $T(n)$-size simulator $S$ such that for every randomized $t(n)$-size distinguisher $D$, we have

$\mid \operatorname{Pr}\left[D\left(X_{n}, Z_{n}\right.\right.$, Out $\left.\left._{V^{*}}\left[P\left(X_{n}, Y_{n}\right) \leftrightarrow V^{*}\left(X_{n}, Z_{n}\right)\right]\right)=1\right]-\operatorname{Pr}\left[D\left(X_{n}, Z_{n}, S\left(X_{n}, Z_{n}\right)\right)=1\right] \mid$ $\leq \epsilon(n)$.

In the above definition, if $L$ is an NP-language, then we require (i.e., assume) $Y_{n}$ to be a witness of $X_{n}$ (this also applies to the corresponding definition below). Weak distributional $(T, t, \epsilon)$-zero-knowledge can be defined in an analogous way by switching the ordering of the quantifiers $\exists S$ and $\forall D$. We now turn to define super-weak distributional $(T, t, \epsilon)$-zero-knowledge.

Definition 6 (super-weak distributional (T,t, $\epsilon$ )-zero-knowledge). Let $(P, V)$ be an interactive proof system for a language $L$. We say that $(P, V)$ is 
super-weak distributional $(T, t, \epsilon)$-zero-knowledge if for every $n \in \mathbb{N}$, every joint distribution $\left(X_{n}, Y_{n}, Z_{n}\right)$ over $\left(L \cap\{0,1\}^{n}\right) \times\{0,1\}^{*} \times\{0,1\}^{*}$, every randomized $t(n)$-size adversary $V^{*}$, and every randomized $t(n)$-size distinguisher $D$, there exists a randomized $T(n)$-size simulator $S$ such that

$$
\begin{aligned}
& \operatorname{Pr}\left[D\left(X_{n}, Z_{n}, \text { Out }_{V^{*}}\left[P\left(X_{n}, Y_{n}\right) \leftrightarrow V^{*}\left(X_{n}, Z_{n}\right)\right]\right)=1\right]-\operatorname{Pr}\left[D\left(X_{n}, Z_{n}, S\left(X_{n}, Z_{n}\right)\right)=1\right] \\
& \leq \epsilon(n) .
\end{aligned}
$$

We may consider an even weaker notion of super-weak distributional zeroknowledge - let us refer to it as super-weak ${ }^{*}$ distributional zero-knowledge - where we only require indistinguishability to hold against deterministic distinguishers $D$ that may output a real value in $[0,1]$ (such a distinguisher can easily be converted to a randomized distinguisher by simply first computing the output $p$ of the deterministic one and then sampling a decision bit $b=1$ with probability $p$ ).

We now show that super-weak distributional $(T, t, \epsilon)$-zero-knowledge is equivalent to distributional $(T, t, \epsilon)$-zero-knowledge if we consider all polynomials for $T$ and $t$ and all inverse polynomials for $\epsilon$. In fact, we prove a more general theorem that also describes the loss in the parameters $T, t$, and $\epsilon$.

Theorem 9. Let $(P, V)$ be an interactive proof system for a language $L$, and suppose $(P, V)$ is super-weak distributional $(T, t, \epsilon)$-zero-knowledge. Then, $(P, V)$ is also distributional $\left(T^{\prime}, t^{\prime}, 2 \epsilon\right)$-zero-knowledge, where $t^{\prime}(n)=\Omega(\epsilon(n) \sqrt{t(n)}-n)$ and $T^{\prime}(n)=O\left(\frac{t^{\prime}(n) \ln \left(n+t^{\prime}(n)\right)}{\epsilon(n)^{2}}\right) \cdot T(n)$.

Proof. Let $n \in \mathbb{N}$, let $\left(X_{n}, Y_{n}, Z_{n}\right)$ be any joint distribution over $\left(L \cap\{0,1\}^{n}\right) \times$ $\{0,1\}^{*} \times\{0,1\}^{*}$, and let $V^{*}$ be any $t(n)$-size adversary. It is easy to see that w.l.o.g., we can assume that the length of $Z_{n}$ is always bounded by $t^{\prime}(n)$, and we can remove the absolute value $|\cdot|$ in the definition of distributional $\left(T^{\prime}, t^{\prime}, 2 \epsilon\right)$ zero-knowledge. Thus, it suffices to show the following claim:

Claim. There exists a $T^{\prime}(n)$-size simulator $S$ such that for every $t^{\prime}(n)$-size distinguisher $D$,

$$
\begin{aligned}
& \operatorname{Pr}\left[D\left(X_{n}, Z_{n}, S\left(X_{n}, Z_{n}\right)\right)=1\right]-\operatorname{Pr}\left[D\left(X_{n}, Z_{n}, \text { Out }_{V^{*}}\left[P\left(X_{n}, Y_{n}\right) \leftrightarrow V^{*}\left(X_{n}, Z_{n}\right)\right]\right)=1\right] \\
& \geq-2 \epsilon(n) .
\end{aligned}
$$

We now proceed to showing the above claim. We define a two-player zero-sum game between a "simulator player" Sim and a "distinguisher player" Adv. The

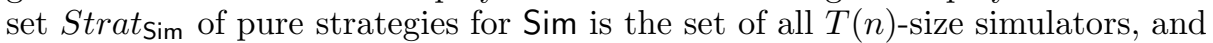
the set Strat $_{\text {Adv }}$ of pure strategies for Adv is the set of all $t^{\prime}(n)$-size distinguishers. The payoff for Sim when Sim chooses a simulator $S \in$ Strat $_{\text {Sim }}$ and Adv chooses a distinguisher $D \in$ Strat $_{\mathrm{Adv}}$ is

$$
\begin{aligned}
& \mu_{n}(S, D) \\
& =\operatorname{Pr}\left[D\left(X_{n}, Z_{n}, S\left(X_{n}, Z_{n}\right)\right)=1\right]-\operatorname{Pr}\left[D\left(X_{n}, Z_{n}, \text { Out }_{V^{*}}\left[P\left(X_{n}, Y_{n}\right) \leftrightarrow V^{*}\left(X_{n}, Z_{n}\right)\right]\right)=1\right] .
\end{aligned}
$$

For mixed strategies (i.e., distributions) $\mathcal{S}$ over $_{S t r a t}$ Sim, and $\mathcal{D}$ over $_{S t r a t} t_{\text {Adv }}$, we define

$$
\mu_{n}(\mathcal{S}, \mathcal{D}):=\mathbb{E}_{S \leftarrow \mathcal{S}, D \leftarrow \mathcal{D}}\left[\mu_{n}(S, D)\right]
$$


The following simple lemma states that any distribution over circuits can be approximated by a small randomized circuit, obtained by taking an appropriate number of samples from the original distribution. This proof technique was used in [1] and 24] for obtaining sparse approximations to randomized strategies in two-player zero-sum games. A fact similar to our lemma was implicitly used by Impagliazzo 22 and several subsequent works, but we find it useful to explicitly formalize it as a lemma (that we hope will be useful also in other contexts).

Lemma 3 (Approximating a distribution over circuits by a small circuit obtained via sampling). Let $X$ and $A$ be finite sets, let $Y$ be any random variable with finite support, let $\mathcal{C}$ be any distribution over s-size randomized circuits of the form $C: X \times \operatorname{Supp}(Y) \rightarrow A$, and let $U$ be any finite set of randomized circuits of the form $u: X \times \operatorname{Supp}(Y) \times A \rightarrow\{0,1\}$. Then, for every $\epsilon>0$, there exists a randomized circuit $\widehat{C}$ of size $T=O\left(\frac{\log |X|+\log |U|}{\epsilon^{2}} \cdot s\right)$ such that for every $u \in U$ and $x \in X$, we have

$$
\left|\mathbb{E}_{C \leftarrow \mathcal{C}}[u(x, Y, C(x, Y))]-\mathbb{E}[u(x, Y, \widehat{C}(x, Y))]\right| \leq \epsilon .
$$

Additionally, there exists a deterministic circuit $\widetilde{C}$ of size $T$ such that for all inputs $x, y, \widetilde{C}(x, y)=\operatorname{Pr}[\widehat{C}(x, y)=1]$.

The lemma follows easily from a Chernoff bound and a union bound; see the full version of this paper for the proof. This proof of the main theorem now follows from three relatively simple steps:

Step 1. We first show that for any mixed strategy $\mathcal{D}$ for Adv (i.e., any distribution over $t^{\prime}(n)$-size distinguishers), there exists a $T(n)$-size simulator $S_{\mathcal{D}} \in$ Strat $_{\text {sim }}$ such that $\mu_{n}\left(S_{\mathcal{D}}, \mathcal{D}\right) \geq-3 \epsilon(n) / 2$. By Lemma 3, we can approximate $\mathcal{D}$ by a $t(n)$-size distinguisher $\widehat{D}$, and then use the super-weak distributional $(T, t, \epsilon)$-zero-knowledge property of $(P, V)$ to get a $T(n)$-size simulator $S_{\widehat{D}}$ for $\widehat{D}$ such that $\mu_{n}\left(S_{\widehat{D}}, \widehat{D}\right) \geq-\epsilon(n)$. Since $\widehat{D}$ approximates $\mathcal{D}$ to within $\epsilon(n) / 2$, we have $\mu_{n}\left(S_{\widehat{D}}, \mathcal{D}\right) \geq-3 \epsilon(n) / 2$, as required.

Step 2. We now apply the minimax theorem to the result of Step 1 to get a mixed strategy $\mathcal{S}$ for $\operatorname{Sim}$ (i.e., a distribution over $T(n)$-size simulators) such that for every $t^{\prime}(n)$-size distinguisher $D \in \operatorname{Strat}_{\text {Adv }}$, we have $\mu_{n}(\mathcal{S}, D) \geq$ $-3 \epsilon(n) / 2$.

Step 3. By Lemma 3 we can approximate $\mathcal{S}$ (from Step 2) by a $T^{\prime}(n)$-size simulator $\widehat{S}$ so that $\mu_{n}(\widehat{S}, D) \geq-2 \epsilon(n)$ for every $t^{\prime}(n)$-size distinguisher $D \in \operatorname{Strat}_{\mathrm{Adv}}$.

The result of Step 3 shows Claim 4, which completes the proof of the theorem. We now provide the details for Steps 1 and 3 .

\section{Details of Step 1.}

By Lemma 3 (in the statement of the lemma, we let $X=\operatorname{Supp}\left(X_{n}\right) \times \operatorname{Supp}\left(Z_{n}\right) \times$ $\{0,1\}^{t^{\prime}(n)}, A=\{0,1\}, Y=0, \mathcal{C}=\mathcal{D}, U$ be a set containing only the circuit $(x, y, a) \mapsto a$, and $\epsilon=\epsilon(n) / 2)$, there exists a distinguisher $\widehat{D}$ of size $O((n+$ 
$\left.\left.t^{\prime}(n)\right)^{2} / \epsilon(n)^{2}\right)=t(n)$ such that for every $x \in X_{n}, z \in Z_{n}$, and $v \in\{0,1\}^{t^{\prime}(n)}$, we have $\left|\operatorname{Pr}_{D \leftarrow \mathcal{D}}[D(x, z, v)=1]-\operatorname{Pr}[\widehat{D}(x, z, v)=1]\right| \leq \epsilon(n) / 2$. Since $(P, V)$ is superweak distributional $(T, t, \epsilon)$-zero-knowledge, there exists a $T(n)$-size simulator $S_{\widehat{D}}$ such that $\mu_{n}\left(S_{\widehat{D}}, \widehat{D}\right) \geq-\epsilon(n)$. From the result above and the definition of $\mu_{n}$, we have $\left|\mu_{n}\left(S_{\widehat{D}}, \mathcal{D}\right)-\mu_{n}\left(S_{\widehat{D}}, \widehat{D}\right)\right| \leq \epsilon(n) / 2$, so $\mu_{n}\left(S_{\widehat{D}}, \mathcal{D}\right) \geq-3 \epsilon(n) / 2$, as required.

\section{Details of Step 3.}

By Lemma 3, there exists a simulator $\widehat{S}$ of size $O\left(\left(\log \left|\operatorname{Strat}_{\mathrm{Adv}}\right| / \epsilon(n)^{2}\right) \cdot T(n)\right)$ such that for every $t^{\prime}(n)$-size distinguisher $D \in$ Strat $_{\text {Adv }}$, we have $\left|\operatorname{Pr}_{S \leftarrow \mathcal{S}}\left[D\left(X_{n}, Z_{n}, S\left(X_{n}, Z_{n}\right)\right)=1\right]-\operatorname{Pr}\left[D\left(X_{n}, Z_{n}, \widehat{S}\left(X_{n}, Z_{n}\right)\right)=1\right]\right| \leq \epsilon(n) / 2$, which implies $\left|\mu_{n}(\mathcal{S}, D)-\mu_{n}(\widehat{S}, D)\right| \leq \epsilon(n) / 2$. Combining this with the result of Step 2, we have $\mu_{n}(\widehat{S}, D) \geq-2 \epsilon(n)$ for every $t^{\prime}(n)$-size distinguisher $D \in$ Strat $_{\text {Adv }}$. Furthermore, the simulator $\widehat{S}$ has size at most $T^{\prime}(n)$, since there are at most $O\left(q(n)+t^{\prime}(n)\right)^{O\left(t^{\prime}(n)\right)}$ circuits of size $t^{\prime}(n)$ on $q(n)$ input bits, so $\mid$ Strat $_{\text {Adv }} \mid \leq$ $O\left(n+t^{\prime}(n)\right)^{O\left(t^{\prime}(n)\right)}$.

We note that by the "additional" part of Lemma 3 , the above proof actually directly shows equivalence also between super-weak* distributional zero-knowledge and distributional zero-knowledge:

Theorem 10. Let $(P, V)$ be an interactive proof system for a language $L$, and suppose $(P, V)$ is super-weak ${ }^{*}$ distributional $(T, t, \epsilon)$-zero-knowledge. Then, $(P, V)$ is also distributional $\left(T^{\prime}, t^{\prime}, 2 \epsilon\right)$-zero-knowledge, where $t^{\prime}(n)=\Omega(\epsilon(n) \sqrt{t(n)}-n)$ and $T^{\prime}(n)=O\left(\frac{t^{\prime}(n) \ln \left(n+t^{\prime}(n)\right)}{\epsilon(n)^{2}}\right) \cdot T(n)$.

\subsection{Laconic Prover Implies Distributional $(T, t, \epsilon)$-Zero-Knowledge}

In this section, we first use Theorem 10 to show that an interactive proof with short prover communication complexity implies distributional $(T, t, \epsilon)$-zeroknowledge. We then describe applications of this result.

Theorem 11. Let $(P, V)$ be an interactive proof system for a language $L$, and suppose that the prover $P$ has communication complexity $\ell(n)$, i.e., the total length of the messages sent by $P$ is $\ell(n)$, where $n$ is the length of the common input $x$. Then, for every function $t^{\prime}(n) \geq \Omega(n)$ and $\epsilon^{\prime}(n),(P, V)$ is distributional $\left(T^{\prime}, t^{\prime}, \epsilon^{\prime}\right)$-zero-knowledge, where $T^{\prime}(n)=O\left(2^{\ell(n)} \cdot \frac{t^{\prime}(n)^{3} \ln \left(t^{\prime}(n)\right)}{\epsilon^{\prime}(n)^{4}}\right)$.

Proof. By Theorem 10, it suffices to show that $(P, V)$ is super-weak* distributional $\left(T, t, \epsilon^{\prime} / 2\right)$-zero-knowledge, where $t(n)=\Theta\left(\frac{t^{\prime}(n)^{2}}{\epsilon^{\prime}(n)^{2}}\right)$ and $T(n)=O\left(2^{\ell(n)}\right.$.

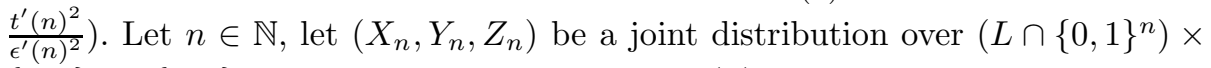
$\{0,1\}^{*} \times\{0,1\}^{*}$, let $V^{*}$ be any randomized $t(n)$-size adversary, and let $D$ be any deterministic $t(n)$-size distinguisher outputting a real value in $[0,1]$. Consider some inputs $x, z$ and randomness $r$ for the verifier $V^{*}$. For any sequence of messages $\left(m_{1}, \ldots, m_{k}\right)$, let $\left(m_{1}, \ldots, m_{k}\right) \leftrightarrow V_{r}^{*}(x, z)$ denote the protocol where 
the prover sends the message $m_{i}$ to $V^{*}$ in round $i$, where the randomness of $V^{*}$ is fixed to $r$.

Let $S$ be the simulator that, on input $(x, z)$ and given randomness $r$, enumerates each of the $2^{\ell(n)}$ possible sequences of messages $\left(m_{1}, \ldots, m_{k}\right)$ of total length $\ell(n)$ (that the prover $P$ may possibly send) and picks the sequence of messages that maximizes $D\left(x, z, O u t_{V^{*}}\left[\left(m_{1}, \ldots, m_{k}\right) \leftrightarrow V_{r}^{*}(x, z)\right]\right)$. By construction it follows that for every random tape $r, D\left(x, z\right.$, Out $\left._{V^{*}}\left[P(x) \leftrightarrow V_{r}^{*}(x, z)\right]\right) \leq$ $D\left(x, z, S_{r}(x, z)\right)$ and thus

$$
\operatorname{Pr}\left[D\left(x, z, \text { Out }_{V^{*}}\left[P(x) \leftrightarrow V^{*}(x, z)\right]\right)=1\right]-\operatorname{Pr}[D(x, z, S(x, z))=1] \leq 0 .
$$

Furthermore, we note that the size of the simulator $S$ is $O\left(2^{\ell(n)} \cdot t(n)\right)=T(n)$. Thus, $(P, V)$ is super-weak* distributional $(T, t, 0)$-zero-knowledge, which completes the proof.

Let us now provide a few corollaries of Theorem [11. The first two are new proofs of old theorems (with some new generalizations). The third one is a new result on 2-round zero-knowledge.

Application 1: Leakage Lemma of Gentry-Wichs. Roughly speaking, the "Leakage Lemma" of Gentry-Wichs 16] states that for every joint distribution $(X, \pi(X))$, where $|\pi(x)|=O(\log |x|)$ ( $\pi$ should be thought of as leakage on $X)$, and for every distribution $Y$ that is indistinguishable from $X$, there exists some leakage $\tilde{\pi}$ such that the joint distributions $(X, \pi(X))$ and $(Y, \tilde{\pi}(Y))$ are indistinguishable. We now show that this result follows as a simple corollary of Theorem 11 .

Two distributions $X$ and $Y$ are $(s, \epsilon)$-indistinguishable if every $s$-size circuit $C$ can only distinguish $X$ from $Y$ by at most $\epsilon$, i.e., $\mid \operatorname{Pr}[C(X)=1]-\operatorname{Pr}[C(Y)=$ $1] \mid \leq \epsilon$.

Corollary 1 (The leakage lemma of Gentry-Wichs [16]). Let $(X, \pi(X))$ be any joint distribution, where $|\pi(X)| \leq \ell$. Let $Y$ be any distribution that is $(s, \epsilon)$-indistinguishable from $X$. Then, there exists a joint distribution $(Y, \tilde{\pi}(Y))$ such that $(X, \pi(X))$ and $(Y, \tilde{\pi}(Y))$ are $\left(s^{\prime}, 2 \epsilon\right)$-indistinguishable, where $s^{\prime}=$ $\Omega\left(\sqrt[3]{\frac{\epsilon^{4} \cdot s}{2^{\ell} \cdot \ln (s)}}\right)$

Proof. Let $L=\{0,1\}^{*}$ be the trivial language with the trivial witness relation $R_{L}(x)=\{0,1\}^{*}$. Let $(P, V)$ be an interactive proof system for $L$ where the prover $P$, on input a statement $x$ with witness $y$, simply sends the first $\ell$ bits of $y$ to the verifier $V$, who simply always accepts. By Theorem $11,(P, V)$ is distributional $\left(T, s^{\prime}, \epsilon\right)$ zero-knowledge, where $T \leq s / 2$. By considering the statement distribution $X$ with witness distribution $\pi(X)$, it follows that there exists a $T$-size simulator $S$ such that $(X, \pi(X))$ and $(X, S(X))$ are $\left(s^{\prime}, \epsilon\right)$-indistinguishable. Also, $(X, S(X))$ and $(Y, S(Y))$ are $(s / 2, \epsilon)$-indistinguishable, since $X$ and $Y$ are $(s, \epsilon)$-indistinguishable and $T \leq s / 2$. It follows that $(X, \pi(X))$ and $(Y, S(Y))$ are $\left(s^{\prime}, 2 \epsilon\right)$-indistinguishable, so letting $\widetilde{\pi}=S$ yields the result. 
Let us note that our proof of the leakage lemma yields an even stronger statement - namely, we have found an efficient simulator $\tilde{\pi}$; such a version of the leakage lemma was recently established by Jetchev and Pietrzak 23. (As an independent contribution, our proof of Theorem 4 is actually significantly simpler than both the proof of [16] and [23.) Additionally, since our result on zero-knowledge applies also to interactive protocols, we directly also get an interactive version of the leakage lemma.

Application 2: Dense Model Theorem We proceed to show that the dense model theorem (e.g., see 28/3013]) follows as a corollary of Theorem 11 A distribution $R$ is $\delta$-dense in a distribution $X$ if for every $r, \operatorname{Pr}[R=r] \leq \frac{1}{\delta} \operatorname{Pr}[X=$ $r$. Equivalently, $R$ is $\delta$-dense in $X$ if there exists a joint distribution $(X, B(X))$ with $\operatorname{Pr}[B(X)=1] \geq \delta$ such that $R=X \mid(B(X)=1)$. Let $U_{n}$ be the uniform distribution over $\{0,1\}^{n}$.

Corollary 2 (The dense model theorem). Let $X$ be any distribution over $\{0,1\}^{n}$ that is $(s, \epsilon)$-indistinguishable from $U_{n}$, and suppose $R$ is $\delta$-dense in $X$. Then, there exists a distribution $M$ that is $(\delta-2 \epsilon)$-dense in $U_{n}$, and $M$ and $R$ are $\left(s^{\prime}, \frac{2 \epsilon}{\delta}\right)$-indistinguishable, where $s^{\prime}=\Omega\left(\sqrt[3]{\frac{\epsilon^{4} \cdot s}{\ln (s)}}\right)$.

Proof. Since $R$ is $\delta$-dense in $X$, there exists a joint distribution $(X, B(X))$ with $\operatorname{Pr}[B(X)=1] \geq \delta$ such that $R=X \mid(B(X)=1)$. Without loss of generality, we can assume that $B(X)$ is always either 0 or 1 . Let $L=\{0,1\}^{*}$ be the trivial language with the trivial witness relation $R_{L}(x)=\{0,1\}^{*}$. Let $(P, V)$ be an interactive proof system for $L$ where the prover $P$, on input a statement $x$ with witness $y$, simply sends the first bit of $y$ to the verifier $V$, who simply always accepts. By Theorem [11, $(P, V)$ is distributional $\left(T, 2 s^{\prime}, \epsilon\right)$-zero-knowledge, where $T \leq s / 2$. By considering the statement distribution $X$ with witness distribution $B(X)$, it follows that there exists a $T$-size simulator $S$ such that $(X, B(X))$ and $(X, S(X))$ are $\left(2 s^{\prime}, \epsilon\right)$-indistinguishable. Also, $(X, S(X))$ and $\left(U_{n}, S\left(U_{n}\right)\right)$ are $(s / 2, \epsilon)$-indistinguishable, since $X$ and $U_{n}$ are $(s, \epsilon)$-indistinguishable and $T \leq$ $s / 2$. It follows that $(X, B(X))$ and $\left(U_{n}, S\left(U_{n}\right)\right)$ are $\left(2 s^{\prime}, 2 \epsilon\right)$-indistinguishable. Thus, $\operatorname{Pr}\left[S\left(U_{n}\right)=1\right] \geq \delta-2 \epsilon($ since $\operatorname{Pr}[B(X)=1] \geq \delta)$, so $U_{n} \mid\left(S\left(U_{n}\right)=1\right)$ is $(\delta-2 \epsilon)$-dense in $U_{n}$. Also, $X \mid(B(X)=1)$ and $U_{n} \mid\left(S\left(U_{n}\right)=1\right)$ are $\left(s^{\prime}, 2 \epsilon / \delta\right)$ indistinguishable, so letting $M=U_{n} \mid\left(S\left(U_{n}\right)=1\right)$ yields the result.

Application 3: 2-Round ZK A final corollary of Theorem 11 is that the classic two-round graph non-isomorphism protocol (which is only known to be honest-verifier zero-knowledge) is also distributional $(T, t, \epsilon)$-zero-knowledge for $T(n)=\operatorname{poly}\left(t(n), \frac{1}{\epsilon(n)}\right) 6$ In fact, by using the complete problem for SZK (the class of promise problems having a statistical zero-knowledge proof for an honest verifier) by Sahai and Vadhan [29], we can show that every language in SZK has a 2 -round distributional $(T, t, \epsilon)$-zero-knowledge proof for $T(n)=\operatorname{poly}\left(t(n), \frac{1}{\epsilon(n)}\right)$.

${ }^{6}$ Recall that in the classic GNI protocol the prover sends just a single bit. 
Theorem 12. For every language $L \in S Z K$ and every function $\delta(n) \geq \frac{1}{2^{\text {poly }(n)}}$, there exists a two-round interactive proof $(P, V)$ for $L$ with completeness 1 $\operatorname{negl}(n)$ and soundness error $\delta(n)$ such that for every function $t$ and $\epsilon,(P, V)$ is distributional $(T, t, \epsilon)$-zero-knowledge, where $T(n)=\operatorname{poly}\left(\frac{1}{\delta(n)}, t(n), \frac{1}{\epsilon(n)}\right)$.

Proof. From [29], there exists a two-round interactive proof $\left(P^{\prime}, V^{\prime}\right)$ for a complete problem $L_{S Z K}$ for $S Z K$ with completeness negligibly close to 1 and soundness error negligibly close to $\frac{1}{2}$, and the prover $P^{\prime}$ only sends a single bit to the verifier $V^{\prime}$. By repeating the proof in parallel $O\left(\log \frac{1}{\delta(n)}\right)$ times, we get a tworound interactive proof for $L_{S Z K}$ with completeness negligibly close to 1 and soundness error $\delta(n)$, and the prover only sends $O\left(\log \frac{1}{\delta(n)}\right)$ bits to the verifier. Then, by Theorem 11, this interactive proof for $L_{S Z K}$ is distributional $(T, t, \epsilon)$ zero-knowledge, where $T(n)=\operatorname{poly}\left(\frac{1}{\delta(n)}, t(n), \frac{1}{\epsilon(n)}\right)$. Since $L_{S Z K}$ is a complete problem for $S Z K$, the theorem follows.

In Theorem 12, if we choose $\delta(n)=\frac{1}{n^{\log n}}, t(n)=n^{\log n}$, and $\epsilon(n)=\frac{1}{n^{\log n}}$, then every language in SZK has a 2-round "quasi-polynomial-time simulatable" distributional zero-knowledge proof (i.e., $T(n)$ is a quasi-polynomial) with completeness $1-\operatorname{negl}(n)$ and negligible soundness error. Alternatively, if we choose $\delta(n)=\frac{1}{\operatorname{poly}(n)}, t(n)=\operatorname{poly}(n)$, and $\epsilon(n)=\frac{1}{\operatorname{poly}(n)}$, then every language in SZK has a 2-round "polynomial-time simulatable" $(T, t, \epsilon)$-distributional zeroknowledge proof (i.e., $T(n)$ is a polynomial) with completeness $1-\operatorname{negl}(n)$ and soundness error $\frac{1}{\text { poly(n)}}$.

\subsection{Necessity of Non-Black-Box Simulation}

The universal simulator in Theorem 12 is obtained via Theorem 11] which uses Theorem 9, so the universal simulator makes non-black-box usage of $V^{*}$. We remark that this non-black-box usage is also necessary (assuming standard complexity theoretic assumptions): We will show that black-box simulation techniques cannot be used to demonstrate distributional $(T, t, \epsilon)$-zero-knowledge for 2 -round proof systems for languages that are hard-on-average. Thus, as long as SZK contains a problem that is hard-on-average, our non-black-box techniques are necessary. Let us first give the definition of black-box distributional $(T, t, \epsilon)$ zero-knowledge.

Definition 7 (black-box distributional (T,t, $\epsilon)$-zero-knowledge). Let $(P, V)$ be an interactive proof system for a language $L$. We say that $(P, V)$ is black-box distributional $(T, t, \epsilon)$-zero-knowledge if for every $n \in \mathbb{N}$ and every joint distribution $\left(X_{n}, Y_{n}, Z_{n}\right)$ over $\left(L \cap\{0,1\}^{n}\right) \times\{0,1\}^{*} \times\{0,1\}^{*}$, there exists a $T(n)$-size simulator $S$ such that for every $t(n)$-size adversary $V^{*}$ and every $t(n)$-size distinguisher $D$, we have

$$
\begin{aligned}
& \mid \operatorname{Pr}\left[D\left(X_{n}, Z_{n}, \text { Out }_{V^{*}}\left[P\left(X_{n}, Y_{n}\right) \leftrightarrow V^{*}\left(X_{n}, Z_{n}\right)\right]\right)=1\right] \\
& -\operatorname{Pr}\left[D\left(X_{n}, Z_{n}, S^{V^{*}\left(X_{n}, Z_{n}\right)}\left(X_{n}, Z_{n}\right)\right)=1\right] \mid \leq \epsilon(n) .
\end{aligned}
$$

where $S^{V^{*}\left(X_{n}, Z_{n}\right)}$ means that $S$ is given oracle access to the verifier $V^{*}\left(X_{n}, Z_{n}\right)$. 
For any language $L$ and any $x \in\{0,1\}^{*}$, let $L(x)=1$ if $x \in L$, and $L(x)=0$ otherwise. We now show that any 2 -round interactive proof for a language $L$ with "hard-on-average" instances is not black-box distributional zero-knowledge.

Theorem 13. Let $L$ be any language with hard-on-average instances, i.e., there exists an ensemble $\left\{X_{n}\right\}_{n \in \mathbb{N}}$ of distributions $X_{n}$ over $\{0,1\}^{n}$ such that for every non-uniform PPT algorithm $A$ and for sufficiently large $n \in \mathbb{N}$, we have $\operatorname{Pr}\left[A\left(X_{n}\right)=L\left(X_{n}\right)\right] \leq \frac{1}{2}+\epsilon(n)$, where $\epsilon$ is any function such that $\epsilon(n)<\frac{1}{12}$ for sufficiently large $n \in \mathbb{N}$.

Then, there exists a polynomial $t$ such that any 2-round interactive proof $(P, V)$ for $L$ with completeness $\frac{2}{3}$ and soundness error at most $\frac{1}{3}$ is not black-box $(T, t, \epsilon)$-distributional zero-knowledge for any polynomial $T$.

Proof. Let $t(n)=O\left(T_{V}(n)\right)$, where $T_{V}(n)$ is a polynomial bound on the running time of $V$ on instances $x$ of length $n$. To obtain a contradiction, suppose $(P, V)$ is black-box $(T, t, \epsilon)$-distributional zero-knowledge for some polynomial $T$. Let $n \in \mathbb{N}$, let $X_{n}^{\prime}$ be $X_{n}$ conditioned on the event $X_{n} \in L$, let $X_{n}^{\prime \prime}$ be $X_{n}$ conditioned on the event $X_{n} \notin L$, let $Y_{n}$ always be the empty string, and let $Z_{n}$ be the uniform distribution over $\{0,1\}^{t(n)}$. Then, there exists a polynomial-size simulator $S$ such that for every $t(n)$-size adversary $V^{*}$ and every $t(n)$-size distinguisher $D$, we have

$$
\begin{aligned}
& \mid \operatorname{Pr}\left[D\left(X_{n}^{\prime}, Z_{n}, \text { Out }_{V^{*}}\left[P\left(X_{n}^{\prime}, Y_{n}\right) \leftrightarrow V^{*}\left(X_{n}^{\prime}, Z_{n}\right)\right]\right)=1\right] \\
& -\operatorname{Pr}\left[D\left(X_{n}^{\prime}, Z_{n}, S^{V^{*}\left(X_{n}^{\prime}, Z_{n}\right)}\left(X_{n}^{\prime}\right)\right)=1\right] \mid \leq \epsilon(n) .
\end{aligned}
$$

Let $V^{*}$ be the verifier that, on input $(x, z)$, runs the honest verifier $V_{z}(x)$ with random tape $z$ to interact with the prover, and then outputs the message $a$ received from the prover. Let $D$ be the distinguisher that, on input $(x, z, a)$, outputs 1 if $V_{z}(x, a)=1$, and 0 otherwise, where $V_{z}(x, a)$ represents the output of $V(x)$ with random tape $z$ and with message $a$ received from the prover.

Claim. $\operatorname{Pr}\left[D\left(X_{n}^{\prime}, Z_{n}, S^{*}\left(X_{n}^{\prime}, Z_{n}\right)\left(X_{n}^{\prime}\right)\right)=1\right] \geq \frac{2}{3}-\epsilon(n)$.

Proof (of claim). Since $(P, V)$ has completeness $\frac{2}{3}$, we have

$$
\begin{aligned}
& \operatorname{Pr}\left[D\left(X_{n}^{\prime}, Z_{n}, \text { Out }_{V^{*}}\left[P\left(X_{n}^{\prime}, Y_{n}\right) \leftrightarrow V^{*}\left(X_{n}^{\prime}, Z_{n}\right)\right]\right)=1\right] \\
= & \operatorname{Pr}\left[\text { Out }_{V}\left[P\left(X_{n}^{\prime}, Y_{n}\right) \leftrightarrow V\left(X_{n}^{\prime}\right)\right]=1\right] \\
\geq & \frac{2}{3} .
\end{aligned}
$$

Now, combining this with (1), we have

$$
\operatorname{Pr}\left[D\left(X_{n}^{\prime}, Z_{n}, S^{V^{*}\left(X_{n}^{\prime}, Z_{n}\right)}\left(X_{n}^{\prime}\right)\right)=1\right] \geq \frac{2}{3}-\epsilon(n),
$$

as required. This completes the proof of the claim.

Claim. $\operatorname{Pr}\left[D\left(X_{n}^{\prime \prime}, Z_{n}, S^{V^{*}\left(X_{n}^{\prime \prime}, Z_{n}\right)}\left(X_{n}^{\prime \prime}\right)\right)=0\right] \geq \frac{2}{3}-\epsilon(n)$. 
Proof (of claim). To obtain a contradiction, suppose $\operatorname{Pr}\left[D\left(X_{n}^{\prime \prime}, Z_{n}, S^{V^{*}\left(X_{n}^{\prime \prime}, Z_{n}\right)}\left(X_{n}^{\prime \prime}\right)\right)=0\right]<\frac{2}{3}-\epsilon(n)$. We note that the event $D\left(X_{n}^{\prime \prime}, Z_{n}, S^{V^{*}}\left(X_{n}^{\prime \prime}, Z_{n}\right)\left(X_{n}^{\prime \prime}\right)\right)=0$ occurs if and only if the event $V_{Z_{n}}\left(X_{n}^{\prime \prime}, S^{V^{*}\left(X_{n}^{\prime \prime}, Z_{n}\right)}\left(X_{n}^{\prime \prime}\right)\right)=0$ occurs, where $V_{Z_{n}}\left(X_{n}^{\prime \prime}, S^{V^{*}\left(X_{n}^{\prime \prime}, Z_{n}\right)}\left(X_{n}^{\prime \prime}\right)\right)$ represents the output of $V\left(X_{n}^{\prime \prime}\right)$ with random tape $Z_{n}$ and with message $S^{V^{*}\left(X_{n}^{\prime \prime}, Z_{n}\right)}\left(X_{n}^{\prime \prime}\right)$ received from the prover. Thus, we have $\operatorname{Pr}\left[V_{Z_{n}}\left(X_{n}^{\prime \prime}, S^{V^{*}\left(X_{n}^{\prime \prime}, Z_{n}\right)}\left(X_{n}^{\prime \prime}\right)\right)=0\right]<\frac{2}{3}-\epsilon(n)$.

Now, consider an adversarial prover $P^{*}$ that, on input $x$ and upon receiving a message $c$ from the verifier $V$, simulates $S(x)$ while responding to oracle queries with the message $c$, and then sends the output of $S(x)$ to $V$. Now, we note that the event $V_{Z_{n}}\left(X_{n}^{\prime \prime}, S^{*}\left(X_{n}^{\prime \prime}, Z_{n}\right)\left(X_{n}^{\prime \prime}\right)\right)=0$ occurs if and only if the event Out $_{V}\left(P^{*}\left(X_{n}^{\prime \prime}, Y_{n}\right) \leftrightarrow V_{Z_{n}}\left(X_{n}^{\prime \prime}\right)\right)=0$ occurs. Thus, we have

$$
\operatorname{Pr}\left[\operatorname{Out}_{V}\left(P^{*}\left(X_{n}^{\prime \prime}, Y_{n}\right) \leftrightarrow V_{Z_{n}}\left(X_{n}^{\prime \prime}\right)\right)=0\right]<\frac{2}{3}-\epsilon(n),
$$

and since we always have $X_{n}^{\prime \prime} \notin L$, this contradicts the assumption that $(P, V)$ has soundness error at most $\frac{1}{3}$. This completes the proof of the claim.

Now, using the polynomial-size simulator $S$ and the $t(n)$-size distinguisher $D$, we will construct a non-uniform PPT algorithm $A$ that contradicts the assumption that $L$ has hard-on-average instances, i.e., for infinitely many $n \in \mathbb{N}$, we have

$$
\operatorname{Pr}\left[A\left(X_{n}\right)=L\left(X_{n}\right)\right]>\frac{1}{2}+\epsilon(n)
$$

Let $A$ be the non-uniform PPT algorithm that, on input $x \in\{0,1\}^{n}$, samples a uniformly random $z$ from $Z_{n}$, computes $S^{V^{*}(x, z)}(x)$ (while simulating the oracle $V^{*}(x, z)$ for $\left.S(x)\right)$ and outputs $D\left(x, z, S^{V^{*}(x, z)}(x)\right)$. Then, for infinitely many $n \in \mathbb{N}$, we have

$$
\begin{aligned}
& \operatorname{Pr}\left[A\left(X_{n}\right)=L\left(X_{n}\right)\right] \\
= & \operatorname{Pr}\left[D\left(X_{n}, Z_{n}, S^{V^{*}\left(X_{n}, Z_{n}\right)}\left(X_{n}\right)\right)=L\left(X_{n}\right)\right] \\
= & \operatorname{Pr}\left[X_{n} \in L\right] \cdot \operatorname{Pr}\left[D\left(X_{n}^{\prime}, Z_{n}, S^{V^{*}\left(X_{n}^{\prime}, Z_{n}\right)}\left(X_{n}^{\prime}\right)\right)=1\right] \\
& +\operatorname{Pr}\left[X_{n} \notin L\right] \cdot \operatorname{Pr}\left[D\left(X_{n}^{\prime \prime}, Z_{n}, S^{V^{*}\left(X_{n}^{\prime \prime}, Z_{n}\right)}\left(X_{n}^{\prime \prime}\right)\right)=0\right] \\
\geq & \operatorname{Pr}\left[X_{n} \in L\right] \cdot(2 / 3-\epsilon(n))+\operatorname{Pr}\left[X_{n} \notin L\right] \cdot(2 / 3-\epsilon(n)) \\
= & \frac{2}{3}-\epsilon(n),
\end{aligned}
$$

where the inequality follows from the two claims above. This contradicts the assumption that $L$ has hard-on-average instances. This completes the proof.

Acknowledgments. We thank Krzysztof Pietrzak for pointing out a mistake in the parameters in Theorem 11 in an earlier version of this paper. 


\section{References}

1. Althfer, I.: On sparse approximations to randomized strategies and convex combinations. Linear Algebra and its Applications 199(suppl.1), 339-355 (1994)

2. Barak, B.: How to go beyond the black-box simulation barrier. In: FOCS, pp. 106-115 (2001)

3. Barak, B., Sahai, A.: How to play almost any mental game over the net - concurrent composition via super-polynomial simulation. In: FOCS, pp. 543-552 (2005)

4. Barak, B., Shaltiel, R., Wigderson, A.: Computational analogues of entropy. In: Arora, S., Jansen, K., Rolim, J.D.P., Sahai, A. (eds.) RANDOM 2003 and APPROX 2003. LNCS, vol. 2764, pp. 200-215. Springer, Heidelberg (2003)

5. Bitansky, N., Paneth, O.: From the impossibility of obfuscation to a new nonblack-box simulation technique. In: FOCS (2012)

6. Bitansky, N., Paneth, O.: On the impossibility of approximate obfuscation and applications to resettable cryptography. In: STOC (2013)

7. Boneh, D., Naor, M.: Timed commitments. In: Bellare, M. (ed.) CRYPTO 2000. LNCS, vol. 1880, pp. 236-254. Springer, Heidelberg (2000)

8. Chung, K.-M., Kalai, Y.T., Liu, F.-H., Raz, R.: Memory delegation. In: Rogaway, P. (ed.) CRYPTO 2011. LNCS, vol. 6841, pp. 151-168. Springer, Heidelberg (2011), http://dl.acm.org/citation.cfm?id=2033036.2033048

9. Chung, K.M., Lui, E., Pass, R.: Can theories be tested? A cryptographic treatment of forecast testing. In: ITCS, pp. 47-56 (2013)

10. Chung, K.M., Pass, R., Seth, K.: Non-black-box simulation from one-way functions and applications to resettable security. In: STOC. ACM (2013)

11. Deng, Y., Goyal, V., Sahai, A.: Resolving the simultaneous resettability conjecture and a new non-black-box simulation strategy. In: 50th Annual IEEE Symposium on Foundations of Computer Science, FOCS 2009, pp. 251-260. IEEE (2009)

12. Dwork, C., Naor, M., Reingold, O., Stockmeyer, L.: Magic functions: In memoriam: Bernard m. dwork 1923-1998. J. ACM 50(6), 852-921 (2003)

13. Dziembowski, S., Pietrzak, K.: Leakage-resilient cryptography. In: 49th FOCS, pp. 293-302. IEEE Computer Society Press (2008)

14. Freund, Y., Schapire, R.E.: Adaptive game playing using multiplicative weights. Games and Economic Behavior 29(1), 79-103 (1999)

15. Frieze, A., Kannan, R.: Quick approximation to matrices and applications. Combinatorica 19(2), 175-220 (1999)

16. Gentry, C., Wichs, D.: Separating succinct non-interactive arguments from all falsifiable assumptions. In: Proceedings of the 43rd Annual ACM Symposium on Theory of Computing, STOC 2011, pp. 99-108. ACM, New York (2011)

17. Goldreich, O.: A uniform-complexity treatment of encryption and zero-knowledge. Journal of Cryptology 6, 21-53 (1993)

18. Goldreich, O., Micali, S., Wigderson, A.: Proofs that yield nothing but their validity or all languages in np have zero-knowledge proof systems. J. ACM 38(3), 690-728 (1991), http://doi.acm.org/10.1145/116825.116852

19. Goldreich, O., Vadhan, S.P., Wigderson, A.: On interactive proofs with a laconic prover. In: Orejas, F., Spirakis, P.G., van Leeuwen, J. (eds.) ICALP 2001. LNCS, vol. 2076, pp. 334-345. Springer, Heidelberg (2001),

http://dl.acm.org/citation. cfm?id=646254.684254

20. Goldwasser, S., Micali, S., Rackoff, C.: The knowledge complexity of interactive proof-systems. In: Proceedings of the Seventeenth Annual ACM Symposium on Theory of Computing, STOC 1985, pp. 291-304. ACM (1985) 
21. Halpern, J., Pass, R.: Game theory with costly computation: formulation and application to protocol security. In: Proceedings of the Behavioral and Quantitative Game Theory: Conference on Future Directions, BQGT 2010, p. 89:1. ACM, New York (2010), http://doi.acm.org/10.1145/1807406.1807495

22. Impagliazzo, R.: Hard-core distributions for somewhat hard problems. In: Proceedings of the 36th Annual Symposium on Foundations of Computer Science, FOCS 1995, pp. 538-545. IEEE Computer Society (1995)

23. Jetchev, D., Pietrzak, K.: How to fake auxiliary input. In: Lindell, Y. (ed.) TCC 2014. LNCS, vol. 8349, pp. 566-590. Springer, Heidelberg (2014)

24. Lipton, R.J., Young, N.E.: Simple strategies for large zero-sum games with applications to complexity theory. In: Proceedings of the Twenty-sixth Annual ACM Symposium on Theory of Computing, STOC 1994, pp. 734-740. ACM (1994)

25. Pass, R.: Bounded-concurrent secure multi-party computation with a dishonest majority. In: STOC 2004. pp. 232-241 (2004)

26. Pass, R., Rosen, A.: Bounded-concurrent secure two-party computation in a constant number of rounds. In: FOCS, pp. 404-413 (2003)

27. Pass, R., Rosen, A.: New and improved constructions of non-malleable cryptographic protocols. In: STOC 2005, pp. 533-542 (2005)

28. Reingold, O., Trevisan, L., Tulsiani, M., Vadhan, S.: Dense subsets of pseudorandom sets. In: Proceedings of the 2008 49th Annual IEEE Symposium on Foundations of Computer Science, FOCS 2008, pp. 76-85 (2008)

29. Sahai, A., Vadhan, S.: A complete problem for statistical zero knowledge. J. ACM 50(2), 196-249 (2003)

30. Trevisan, L., Tulsiani, M., Vadhan, S.: Regularity, boosting, and efficiently simulating every high-entropy distribution. In: Proceedings of the 2009 24th Annual IEEE Conference on Computational Complexity, CCC 2009, pp. 126-136. IEEE Computer Society (2009)

31. Vadhan, S., Zheng, C.J.: A uniform min-max theorem with applications in cryptography. In: Canetti, R., Garay, J.A. (eds.) CRYPTO 2013, Part I. LNCS, vol. 8042, pp. 93-110. Springer, Heidelberg (2013)

32. Yao, A.C.: Theory and application of trapdoor functions. In: Proceedings of the 23rd Annual Symposium on Foundations of Computer Science, SFCS 1982, pp. 80-91 (1982) 